\title{
The Effect of Scale on the Mechanical Properties of Jointed Rock Masses
}

Francois E. Heuze

DTRA Advanced Schoolhouse Course, Springfield, VA, June 14-15, 2004

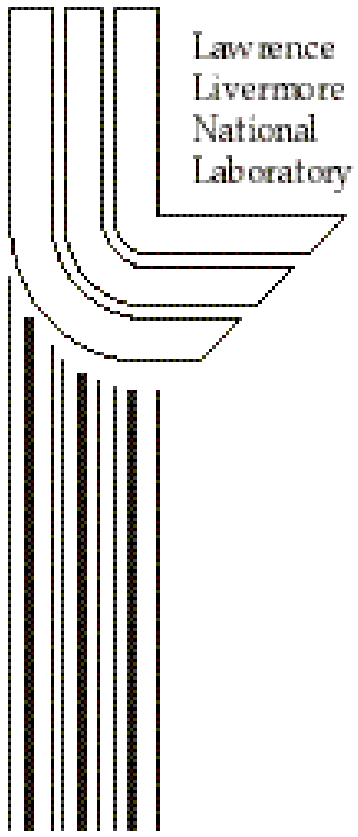

May, 2004 


\section{DISCLAIMER}

This document was prepared as an account of work sponsored by an agency of the United States Government. Neither the United States Government nor the University of California nor any of their employees, makes any warranty, express or implied, or assumes any legal liability or responsibility for the accuracy, completeness, or usefulness of any information, apparatus, product, or process disclosed, or represents that its use would not infringe privately owned rights. Reference herein to any specific commercial product, process, or service by trade name, trademark, manufacturer, or otherwise, does not necessarily constitute or imply its endorsement, recommendation, or favoring by the United States Government or the University of California. The views and opinions of authors expressed herein do not necessarily state or reflect those of the United States Government or the University of California, and shall not be used for advertising or product endorsement purposes.

Work performed under the auspices of the U. S. Department of Energy by the University of California Lawrence Livermore National Laboratory under Contract W-7405-Eng-48.

This report has been reproduced directly from the best available copy.

Available to DOE and DOE contractors from the

Office of Scientific and Technical Information

P.O. Box 62, Oak Ridge, TN 37831

Prices available from (423) 576-8401

http: / / apollo.osti.gov/bridge/

Available to the public from the National Technical Information Service

U.S. Department of Commerce 5285 Port Royal Rd., Springfield, VA 22161 http:// www.ntis.gov/

OR

Lawrence Livermore National Laboratory Technical Information Department's Digital Library http:/ / www.llnl.gov/tid/Library.html 


\begin{abstract}
These notes were prepared for presentation at the Defense Threat Reduction Agency's (DTRA) Hard Target Research and Analysis Center (HTRAC), at the occasion of a short course held on June 14-15, 2004.

The material is intended for analysts who must evaluate the geo-mechanical characteristics of sites of interest, in order to provide appropriate input to calculations of ground shock effects on underground facilities in rock masses. These analysts are associated with the Interagency Geotechnical Assessment Team (IGAT).
\end{abstract}

Because geological discontinuities introduce scale effects on the mechanical properties of rock formations, these large-scale properties cannot be estimated on the basis of tests on small cores.

Accordingly, the outline of the lecture is as follows:

- Geological discontinuities

- effect on ground shock

- effect on failure of underground structures

- basic mechanical properties

- scale effects

- Rock Masses

- deformability of rock masses

- scale effects

- strength of rock masses

- scale effects

- geological strength index (GSI)

- References 


\title{
THE EFFECT OF SCALE ON THE MECHANICAL PROPERTIES OF JOINTED ROCK MASSES
}

\author{
Francois E. Heuze \\ LLNL, Livermore, CA

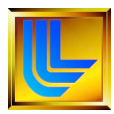 \\ DTRA Advanced Schoolhouse Course \\ Springfield, VA \\ June 14-15, 2004
}

\section{Outline}

Geological discontinuities

- effect on ground shock

- effect on failure of underground structures

- basic mechanical properties

- scale effects

Rock Masses

- deformability of rock masses

- scale effects

- strength of rock masses

- scale effects

- geological strength index (GSI)

References 


\section{Geological discontinuities}

\section{Effects of rock discontinuities - ground shock}

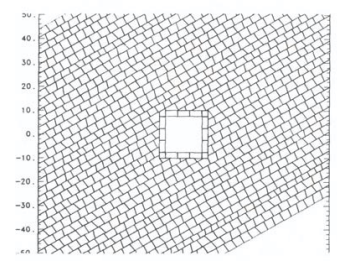

Configuration

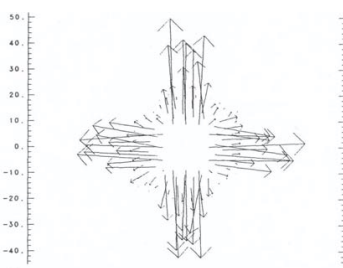

Early time velocity field

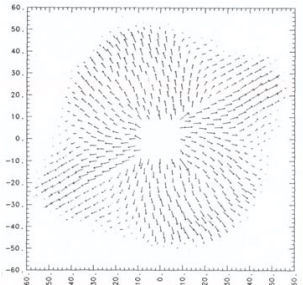

Later time velocities

After Walton et al, 1991.

This ground shock pattern had been observed in "sugar cube" tests by Melzer (1970). 


\section{Effects of rock discontinuities - ground shock}

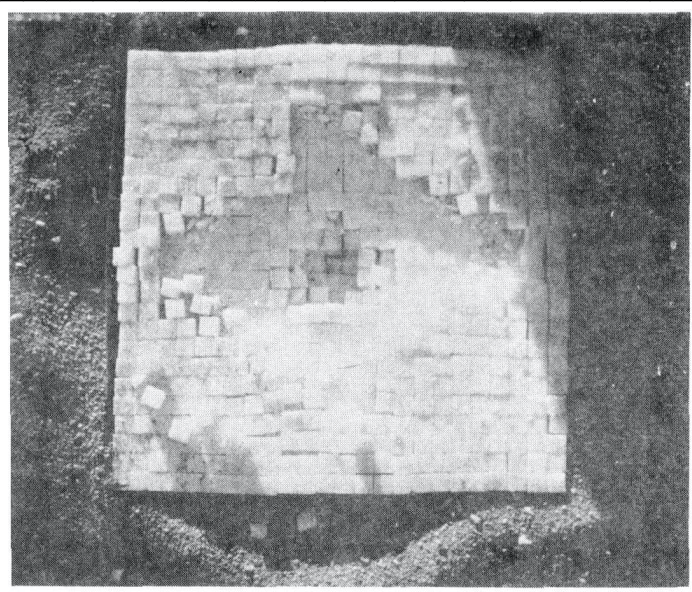

After Melzer (1970). Courtesy of S. Blouin.

\section{Effects of rock discontinuities - ground shock}

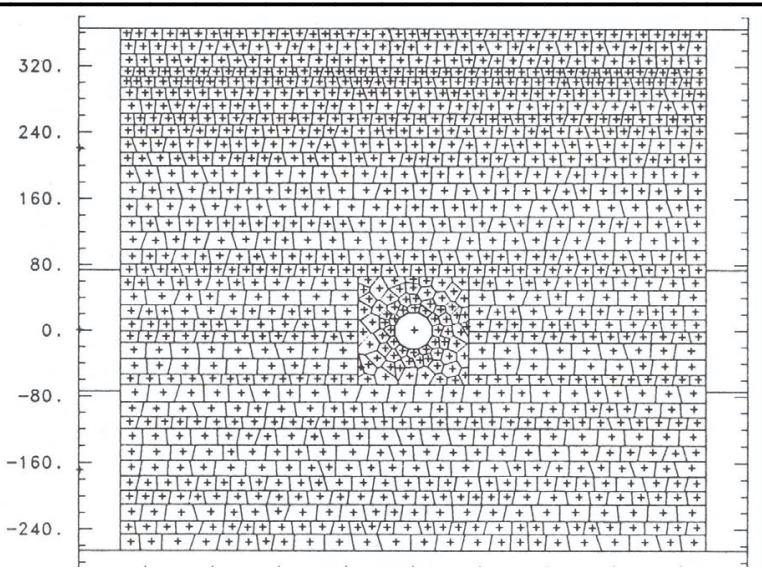

Modeling of a SHOAL-like event (12kt) with the DIBS discrete element code (after Heuze et al, 1993). 


\section{Effects of rock discontinuities - ground shock}

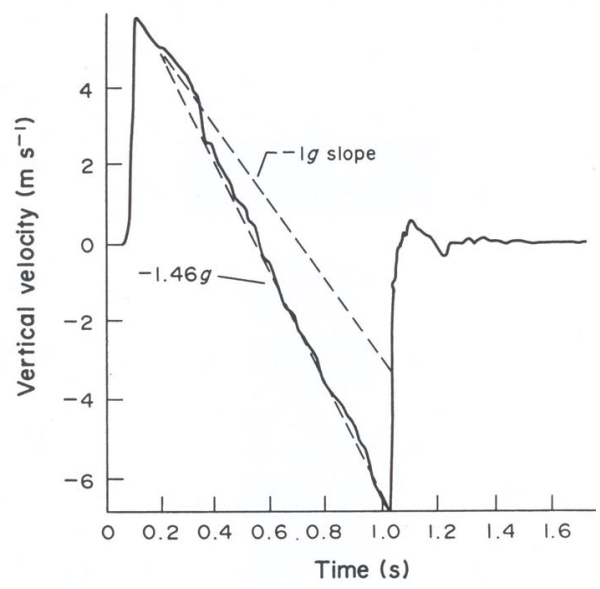

(after Heuze et al, 1993)

The DIBS modeling of a SHOAL-like event showed for the first time a surface spall return acceleration well in excess of $1 \mathrm{~g}$, as had been observed in SHOAL.

\section{Effects of rock discontinuities - ground shock}

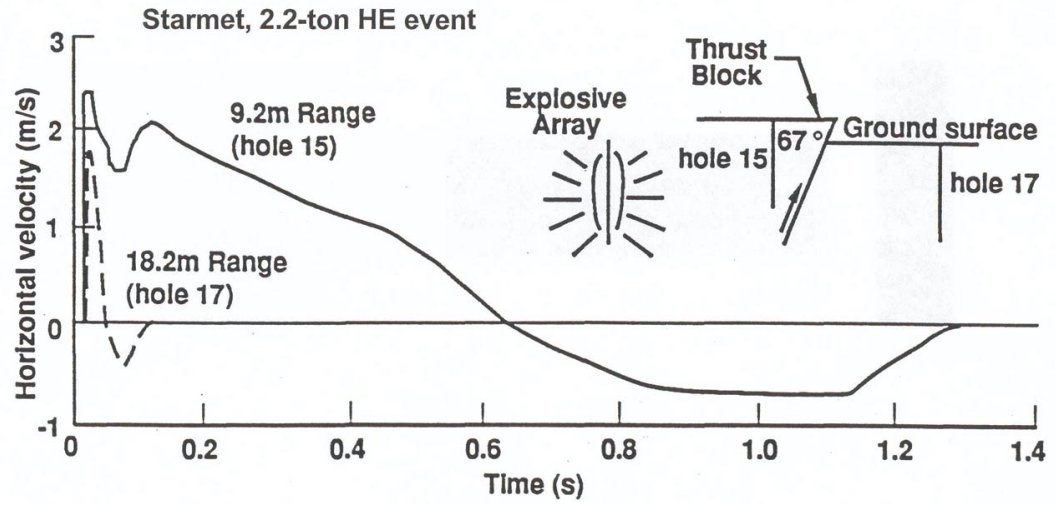

STARMET HE event in granite, NM (Blouin and Kaiser, 1972). Note the very large influence of a geologic discontinuity on the displacement field. 


\section{Effects of rock discontinuities - ground shock}
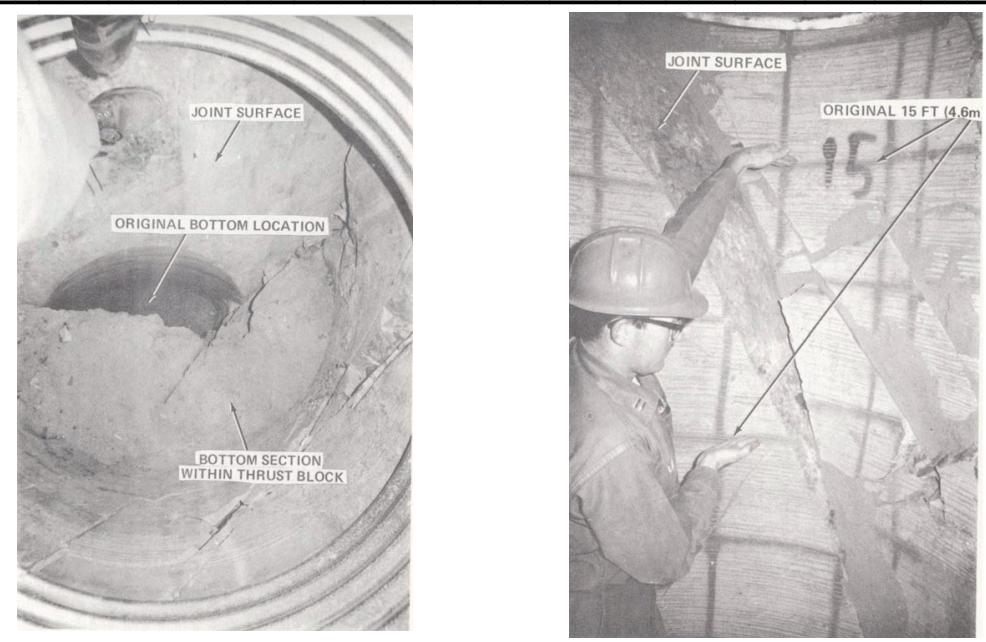

Model missile silos in the STARMET event (Blouin and Kaiser, 1972)

Effects of rock discontinuities - ground shock

Tunnel in tuff, Nevada Test Site

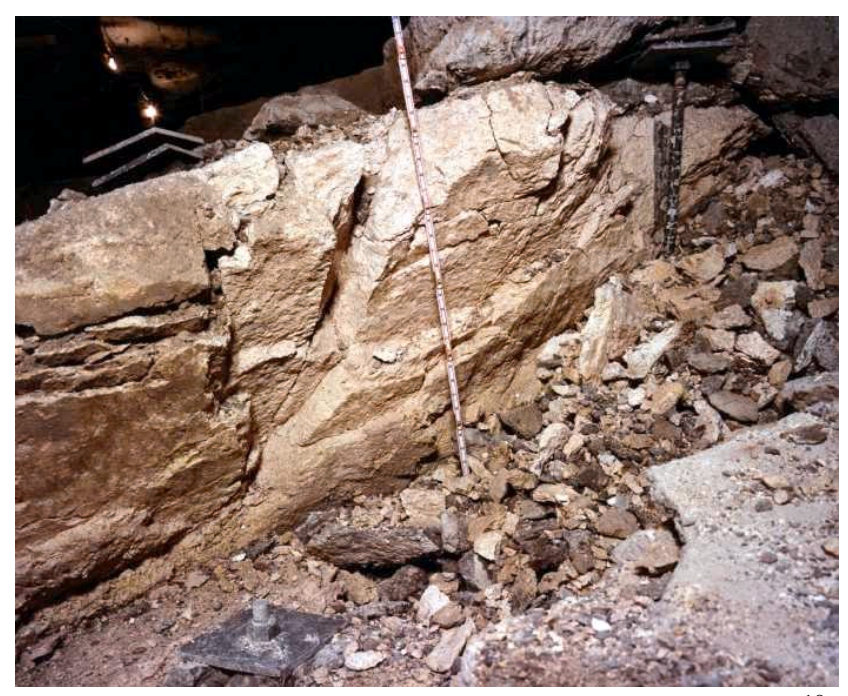


Effects of rock discontinuities - ground shock

Tunnel in tuff, Nevada Test Site

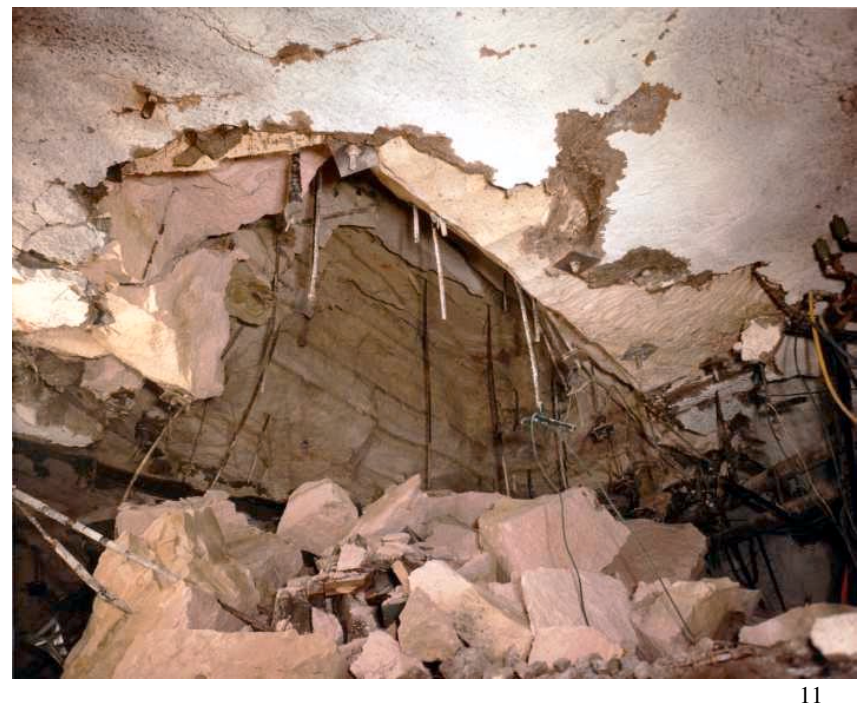

Effects of rock discontinuities - slopes

In granite, near

Tioga Pass, CA

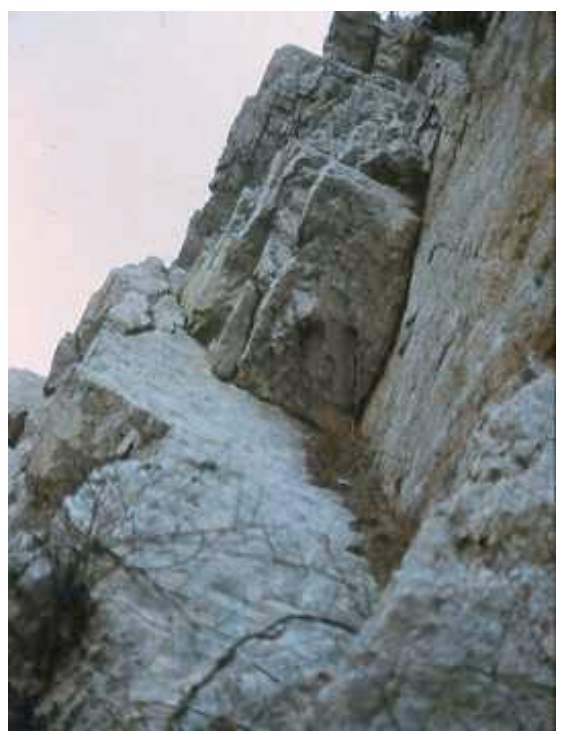




\section{Effects of rock discontinuities - slopes}

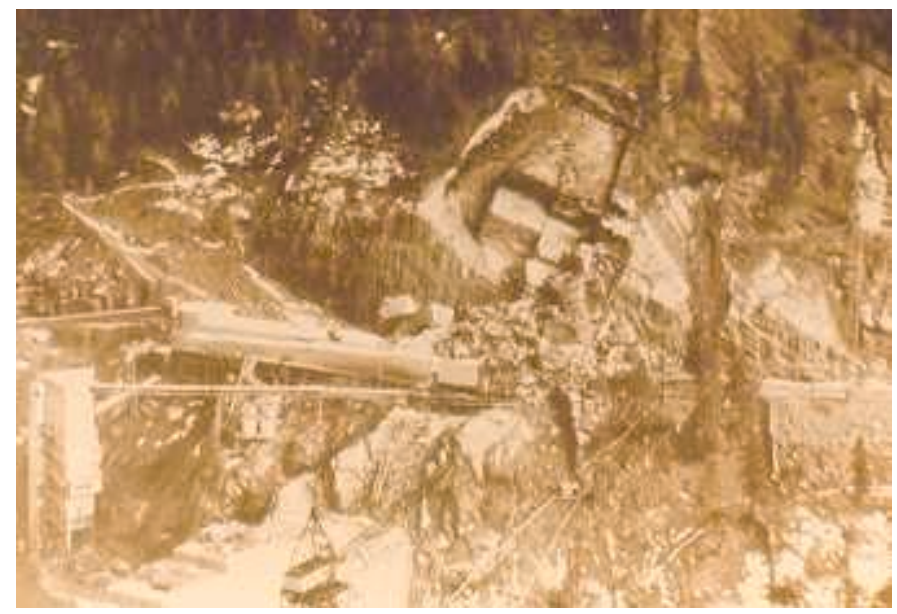

Near Libby Dam, MT (courtesy D. Lachel)

Effects of rock discontinuities - coal mines

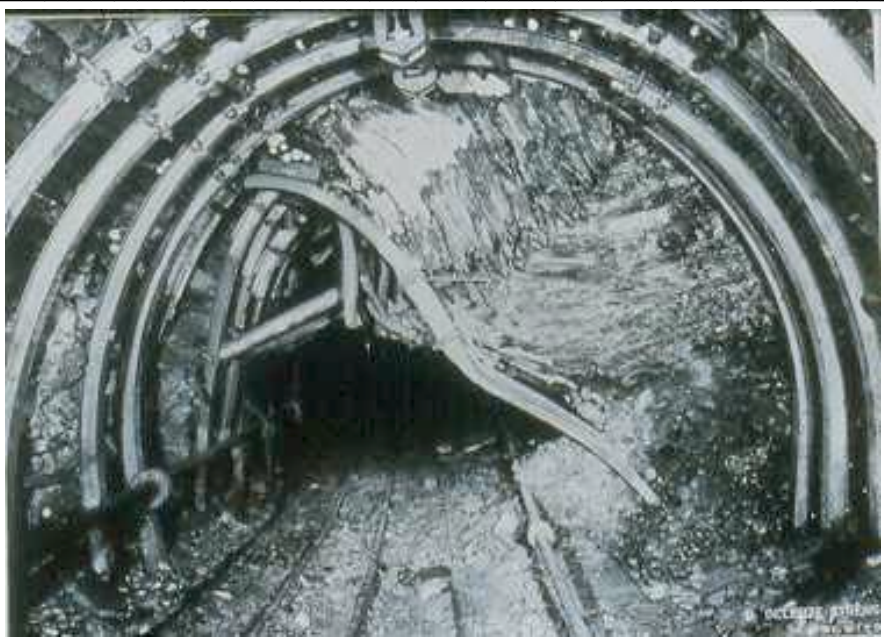

Ground failure in a Belgian coal mine after a coal bump 


\section{Tunnel failure kinematics}
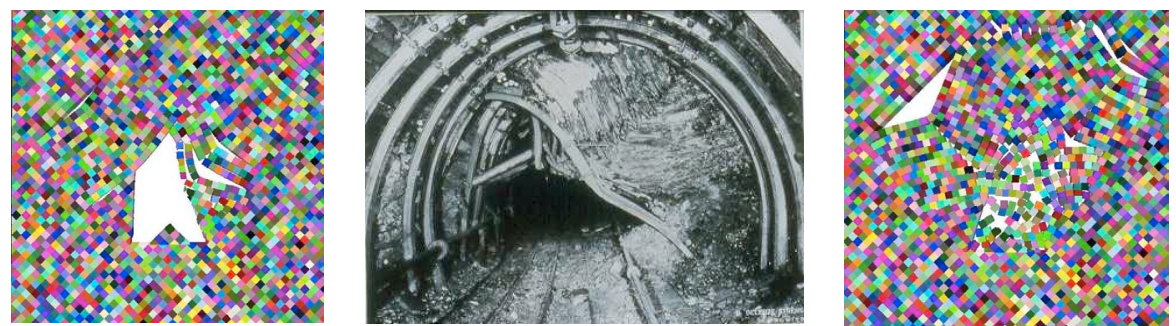

Simulations that discretely include geological discontinuities are required to model the mechanics of failure of tunnels in jointed rocks. Generic discrete element calculations are shown for illustration (Heuze, 2004).

\section{Tunnel failure kinematics (cont.)}

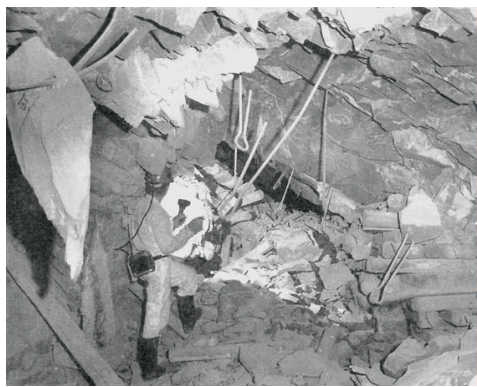

Damaged gold mine entry after a rock burst

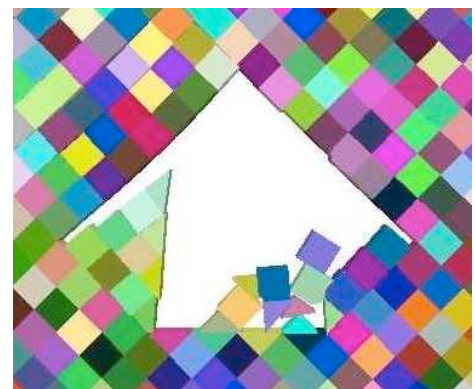

Generic LDEC calculation

(Heuze, 2004) 


\section{Tunnel failure kinematics}

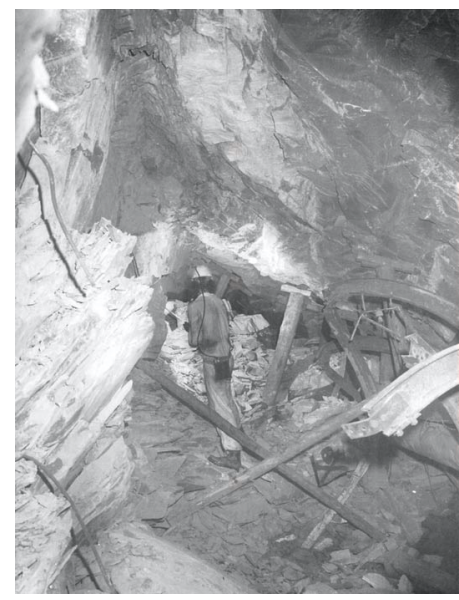

Damaged gold mine entry after a rockburst

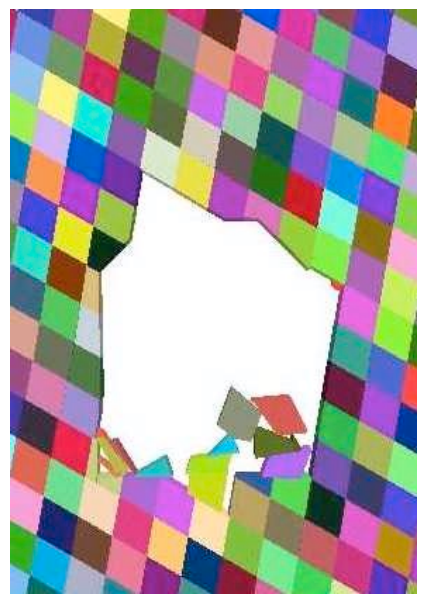

Generic LDEC calculation (Heuze, 2004)

\section{Mechanical attributes}

The basic mechanical concept of a "joint":

- Normal stress : $\sigma=$ N/A

- Shear stress $\tau=T / A$

- Shear displacement : $\mathbf{u}$ or $\Delta \mathbf{u}$

- Normal displacement : $\mathbf{v}$ or $\Delta \mathbf{v}$

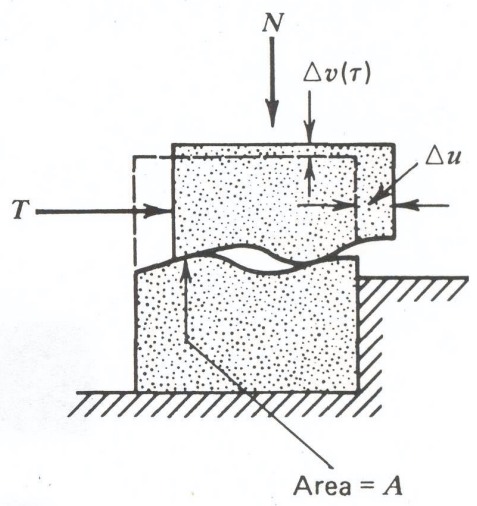

- The mechanical properties of interest under shear stress and normal stress conditions are the stiffnesses (shear and normal), the dilatancy, and the shear strength (Goodman, 1980). 


\section{Behavior of a joint under shear under constant $\sigma$}

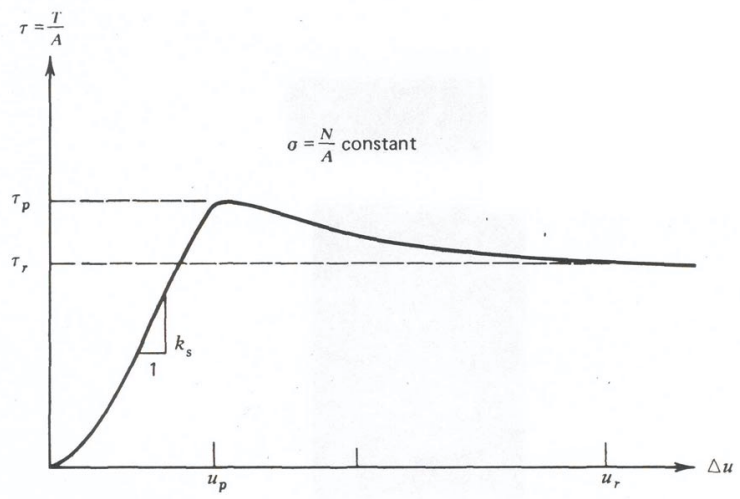

The shear stiffness is Ks. The peak shear strength is $\tau_{p}$ and the residual shear strength is $\tau_{r}$. Rough joints also can dilate during shearing (Goodman, 1980).

\section{Behavior of a joint under compression}

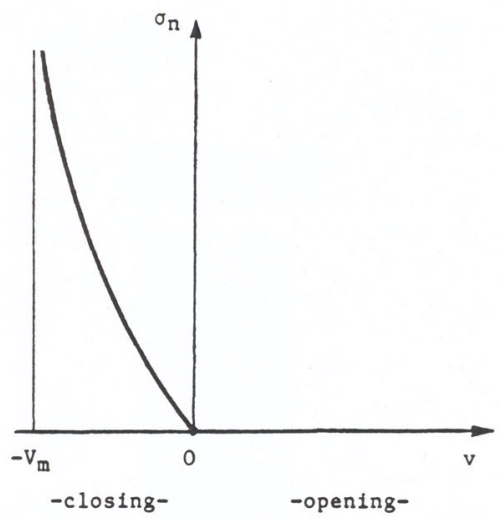

The normal stiffness, $\mathrm{Kn}$, is the slope of the $\mathrm{s}, \mathrm{v}$ curve and the joint has a maximum closure $v_{m}$. Kn increases as the joint closes (Goodman, 1980). 
Shear testing machines with control of normal stress

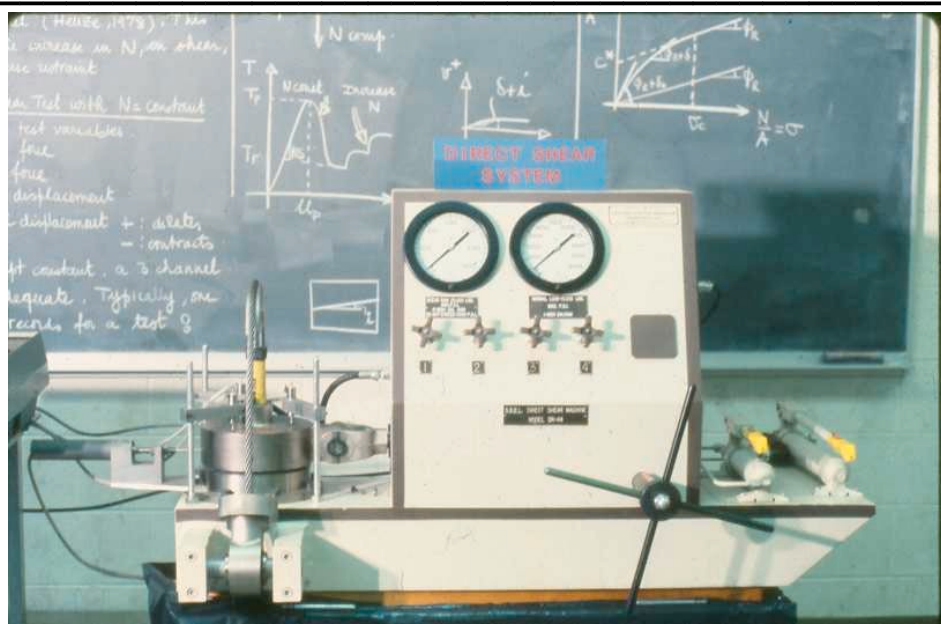

System at C.U. Boulder (1979), after a design by SBEL, Phoenix, AZ

\section{Typical shear stress-deformation behavior}
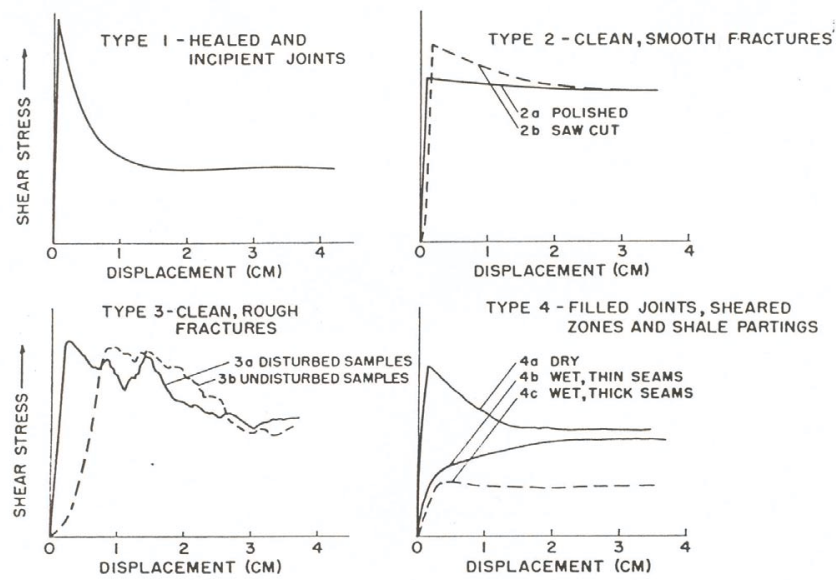

After Goodman, 1970 


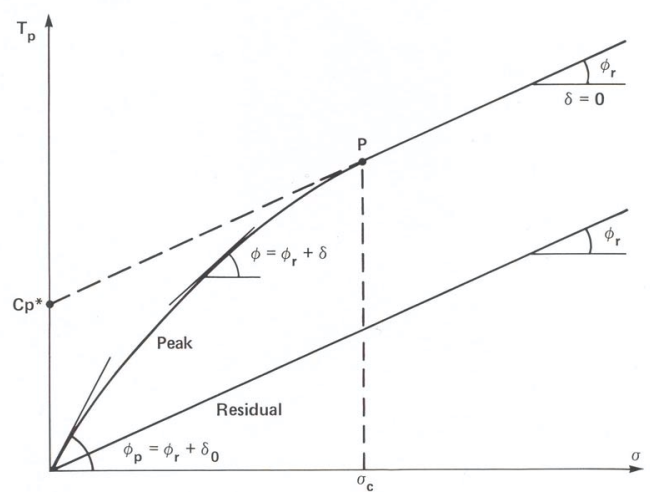

In the $\tau-\sigma$ plane there are two envelopes: one for peak shear strength and one for residual shear strength. Above $\sigma=\sigma_{c}$ there is no dilation in shear.

\section{A perspective on shear strength (N. Barton)}

Barton,s (1973) empirical equation for peak shear strength:

$$
\tau_{p}=\sigma_{n} \tan \left[J R C \log _{10}\left(J C S / \sigma_{n}\right)+\phi_{r}\right]
$$

$\sigma_{\mathrm{n}} \quad:$ normal stress on the joint

JCS : effective joint wall compressive strength (often taken as $\sigma_{\mathrm{c}}$ )

$\sigma_{c}:$ wall rock unconfined compressive strength

JRC : joint roughness coefficient 


\section{A perspective on shear strength (cont.)}

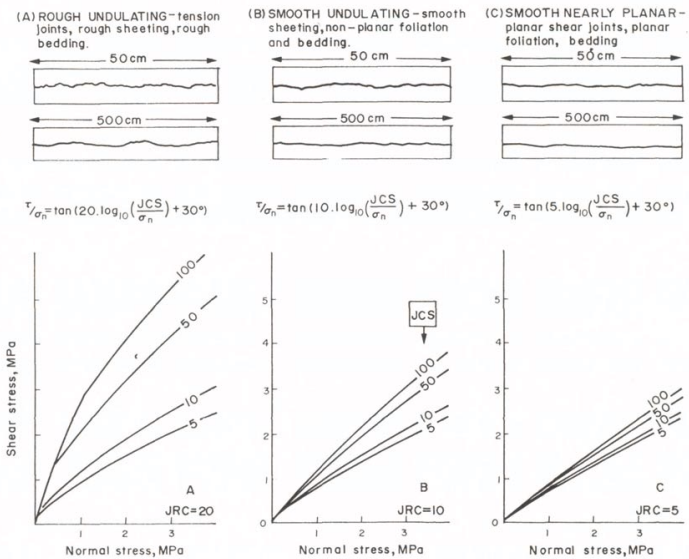

Examples of JRC values and shear strength for different JCS values (Bandis, Lumsden , and Barton, 1981)

\section{Scale effects on shear strength (Bandis et al, 1981)}
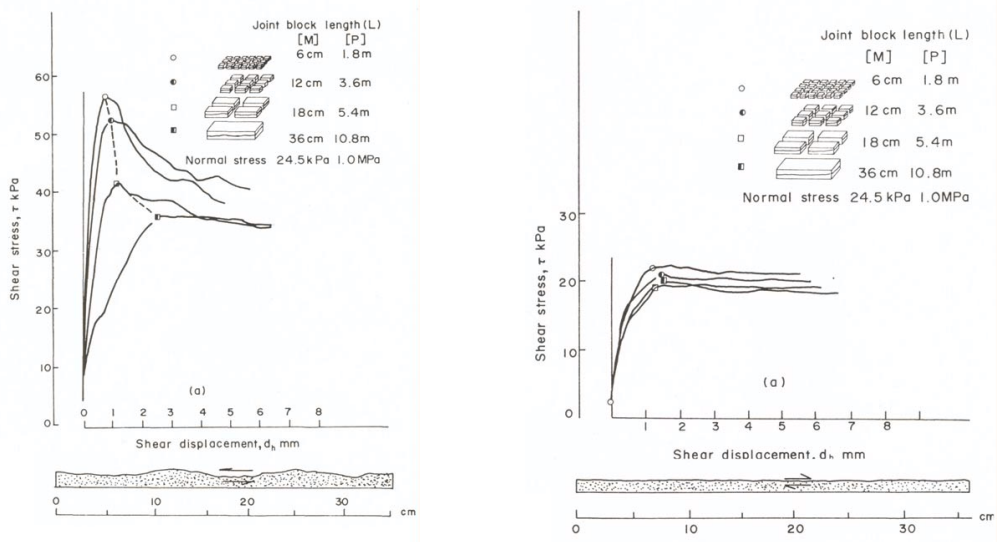

Experimental results

Rough joint: scale effect

Smooth joint: no scale effect 


\section{Scale effects on joint shear strength (cont.)}

Scaling equations proposed by Barton et al, 1985. The subscript $\mathbf{n}$ refers to in-situ. The subscript 0 refers to laboratory.

- Shear displacement to peak shear strength.

$L$ is the sample dimension in meters.

$\delta($ peak $)=\frac{L_{\mathrm{n}}}{500}\left[\frac{\mathrm{JRC}_{\mathrm{N}}}{L_{\mathrm{n}}}\right]^{0.33}$

- Joint Roughness Coefficient

$$
\mathrm{JRC}_{\mathrm{n}}=\mathrm{JRC}_{0}\left[\frac{L_{\mathrm{n}}}{L_{0}}\right]^{-0.02 \mathrm{JRC}_{0}}
$$

- Joint Compressive Strength

$$
\mathrm{JCS}_{\mathrm{n}}=\mathrm{JCS}_{0}\left[\frac{L_{\mathrm{n}}}{L_{0}}\right]^{-0.03 \mathrm{JRC}_{0}}
$$

\section{Scale effects on shear strength (cont.)}

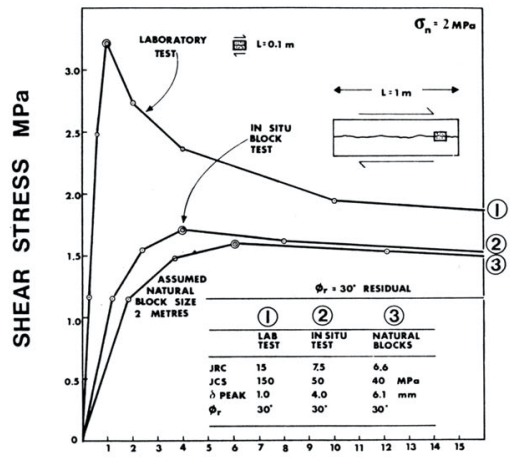

SHEAR DISPLACEMENT $\mathrm{mm}$

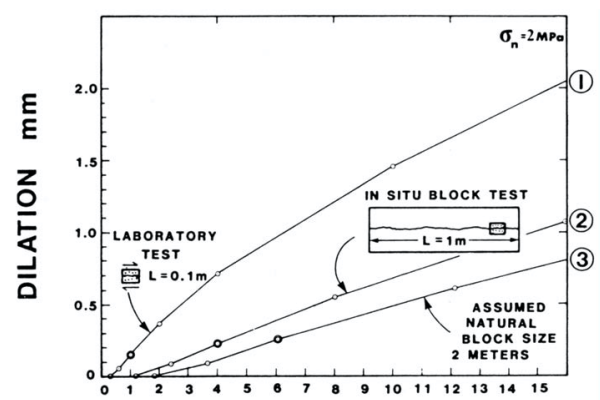

SHEAR DISPLACEMENT $\mathrm{mm}$

Laboratory results vs. expected in-situ results, based on the preceding scaling equations (Barton et al, 1985) 


\section{Scale effects on shear strength, and joint stiffnesses}

$+1 \mathrm{MPa}=2.5 \mathrm{MPa} \triangle 5 \mathrm{MPa}=10 \mathrm{MPa}$

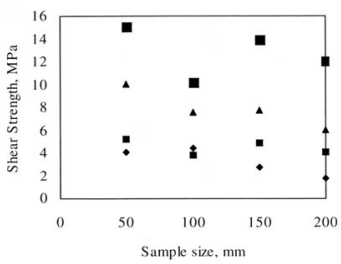

- $1 \mathrm{MPa}$ - 2.5MPa $4 \mathrm{MPa}$-10MPa

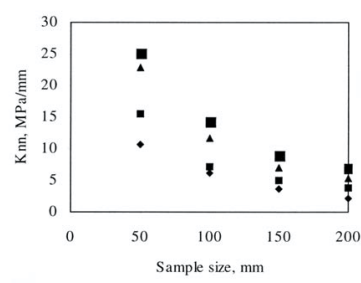

- $1 \mathrm{MPa}$ - 2.5MPa $\triangle \mathrm{MPa}$ —10MPa

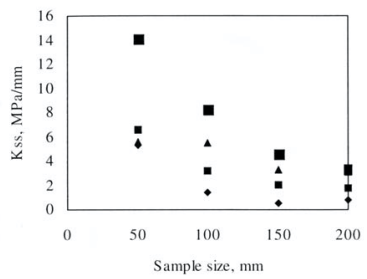

Tests on high-strength concrete replicas of a natural joint in granite, at various sizes and different constant normal stresses.

After Fardin et al., 2003.

\section{Scale effects on normal stiffness; an explanation}

- $1 \mathrm{MPa} \approx 2.5 \mathrm{MPa} \wedge 5 \mathrm{MPa} \square 10 \mathrm{MPa}$
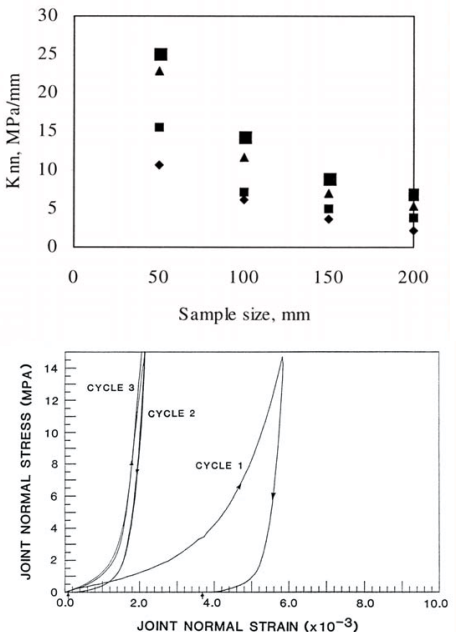

For this figure, Fardin et al. indicate that $\mathrm{Knn}$ was calculated "at the linear part of the third loading cycle".

To explain this procedure, one can look at the results of cyclic normal loading of a sandstone joint reported by J. A. Brown et al. (LANL report BMO-TR-88-06, 1988). 


\section{Deformability of Rock Masses}

\section{Plate tests - Example (Wallace et al, 1970)}

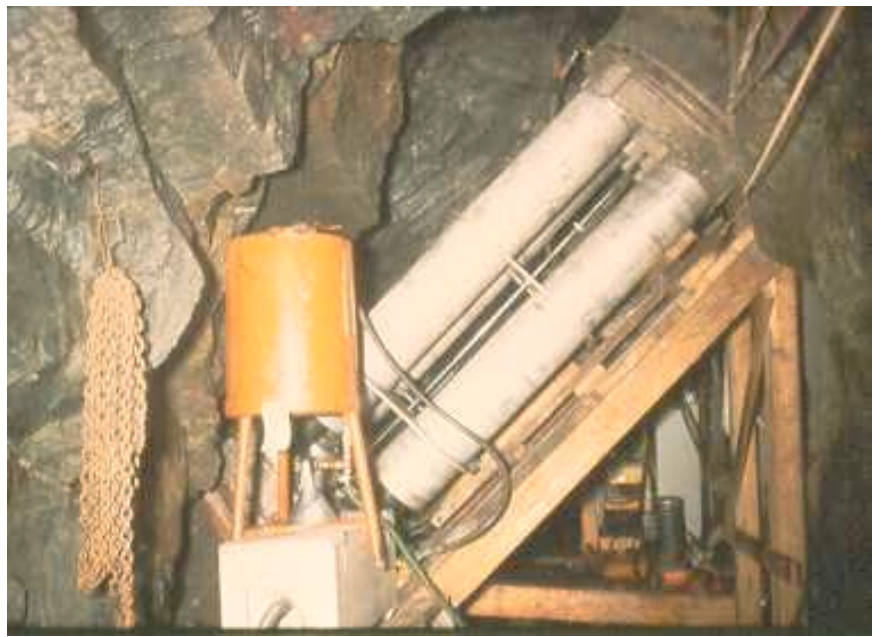

Note: USBR cost, 10 years ago, at Monk Hollow dam site, Utah, was 300K for 6 tests, not including rock surface preparation (G. Scott, pers. communic., 05/08/03) 


\section{Plate test analysis (Belin, 1959)}

In isotropic media, the modulus of the rock mass is calculated as:

$$
E=K \cdot P \cdot \pi \cdot a \cdot\left(1-v^{2}\right) / U
$$

where

- $\mathrm{K}$ : coefficient $=\mathbf{0 . 5 0}$ for a perfectly rigid plate

$$
=0.54 \text { for a perfectly flexible plate }
$$

- P : applied pressure on the plate

- a : radius of the plate (assumed circular)

- $v$ : Poisson,s ratio of the rock mass (assume it to be $\mathbf{0 . 2 5}$ )

- $\mathrm{U}$ : average displacement of the plate

Pressure chamber tests (Wallace et al, 1970)

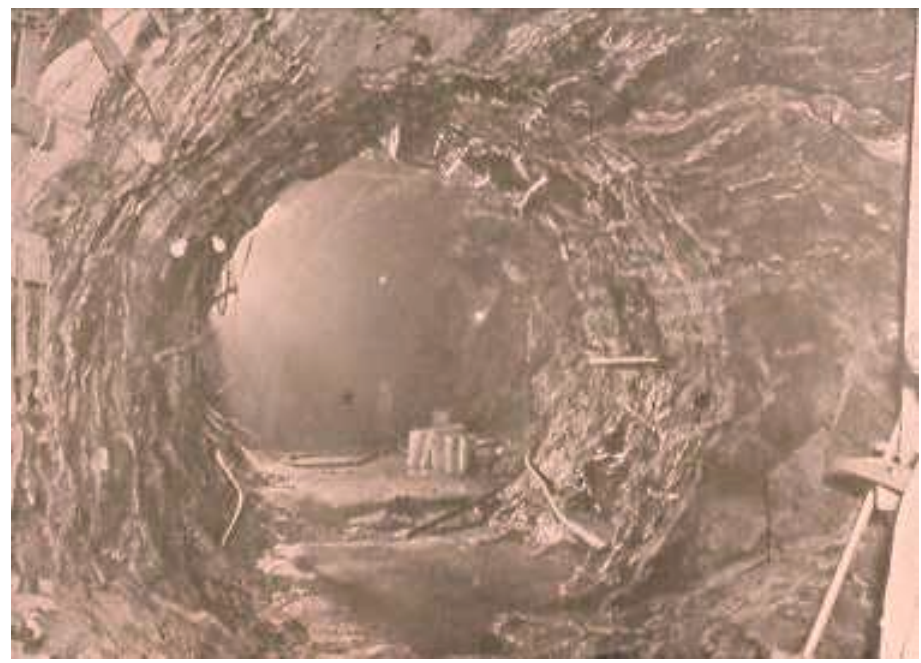


Pressure chamber tests (Wallace et al, 1970)

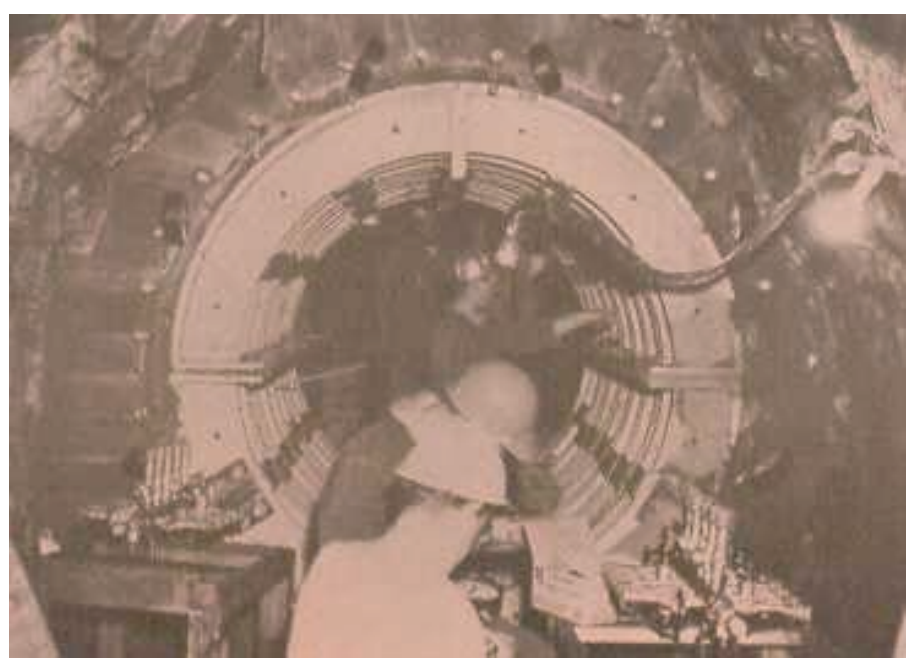

Pressure chamber tests (Wallace et al, 1970)

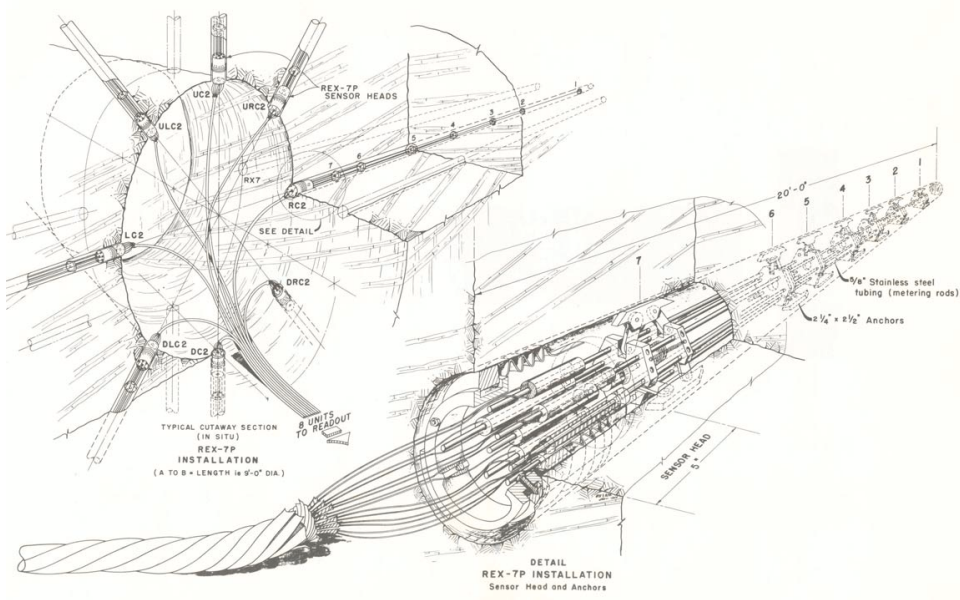




\section{Analysis of pressure tests in circular openings}

This applies to tunnel tests such as above, or to dilatometer tests in boreholes.

Measuring the change in diameter, isotropic case:

$$
E=\Delta P \cdot D \cdot(1+v) / \Delta D
$$

where:

$\Delta P$ : increase in applied pressure

D : diameter

$v$ : Poisson,s ratio of the rock mass (assume 0.25)

$\Delta D$ : change in diameter

or

Measuring the displacement $U(r)$ at depth " $r$ " into the rock mass:

$$
E=\left[\Delta P \cdot D^{2} \cdot(1+v)\right] /[4 \cdot r \cdot U(r)]
$$

The NX-Borehole Jack

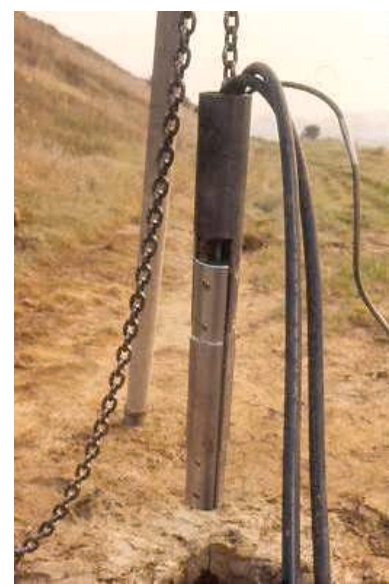

See Goodman et al (1972), and Heuze and Amadei (1985).
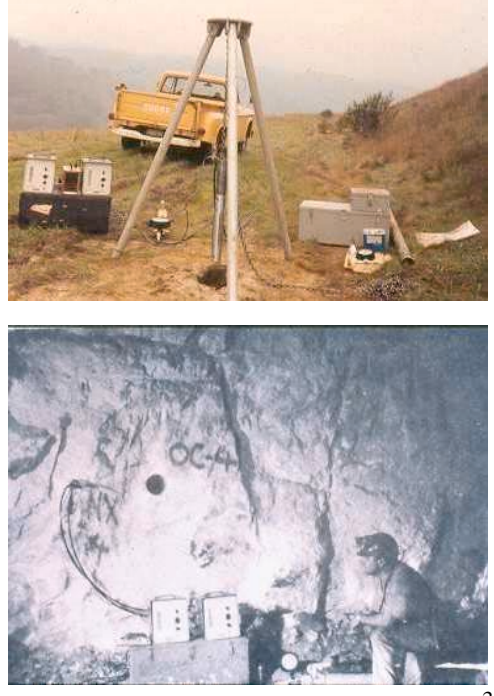


\section{Other field deformability tests - Flat jacks}

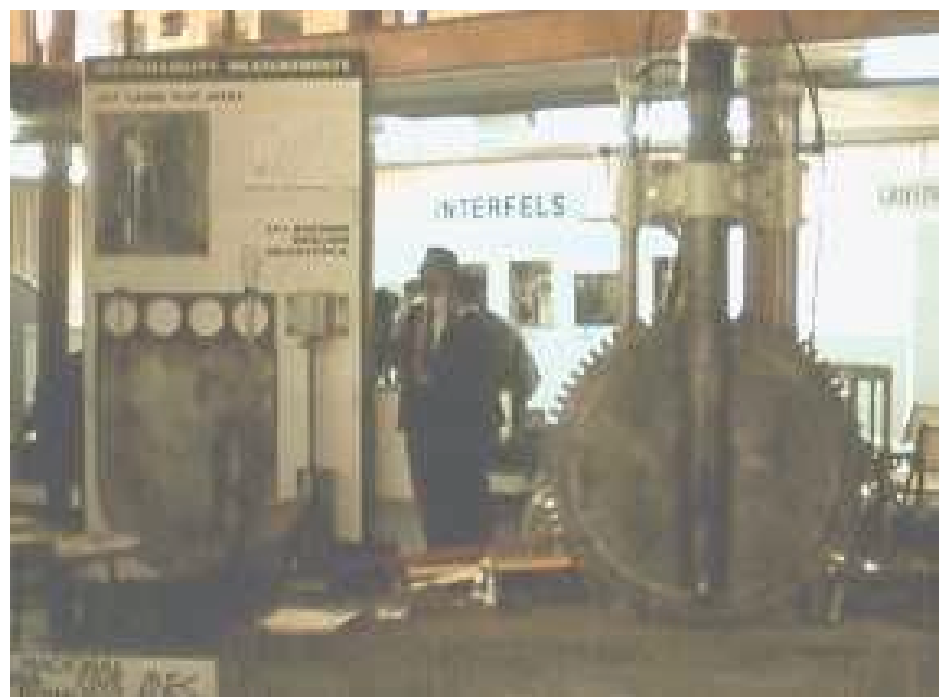

\section{Comparison of different tests - Scale effects}

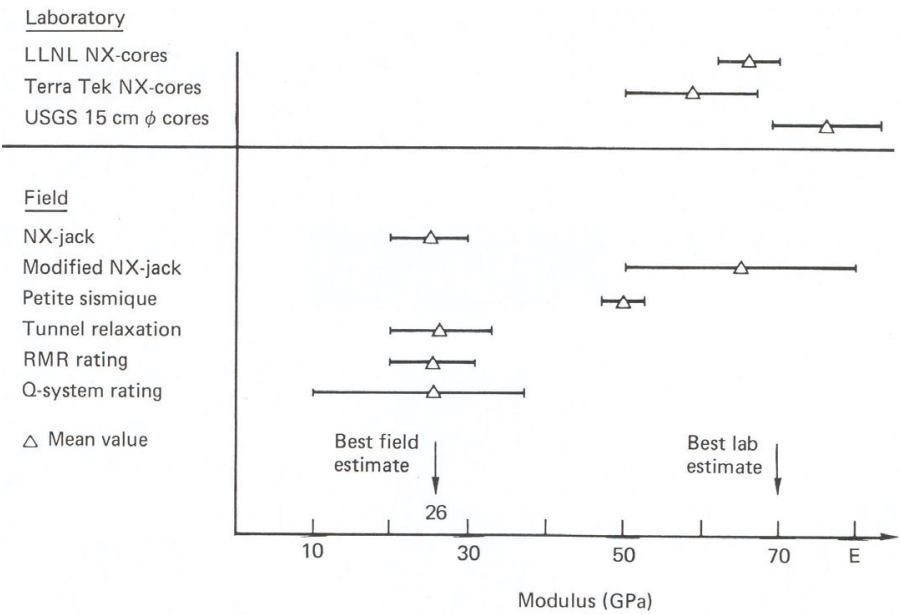

Climax granite, NTS, Nevada, (Heuze et al, 1982) 


\section{Static vs. dynamic moduli; ex: sedimentary rocks}

\begin{tabular}{|c|c|c|c|c|c|c|c|c|}
\hline $\begin{array}{c}\text { Dam } \\
\text { Name }\end{array}$ & Country & Rock & Areq & $\underset{10^{3} \mathrm{~kg} / \mathrm{cm}^{2}}{E_{\text {silom }}}$ & Test & $\underset{\mathrm{kg} / \mathrm{cm}^{2}}{\mathrm{P}}$ & $\begin{array}{c}\text { Eatat } \\
\text { (During Unl.) } \\
10^{x} \mathrm{~kg} / \mathrm{cm}^{2}\end{array}$ & $\frac{E_{\text {nelam }}}{E_{\text {ntat }}}(=:)$ \\
\hline Sylvenstein & Germany & dolomite & $\begin{array}{l}\text { right slope } \\
\text { left slope }\end{array}$ & $\begin{array}{r}850 \\
1100\end{array}$ & jack load & $40-160$ & $71-146$ & $12-5.8$ \\
\hline Limberg & Austria & lam. limest. & $\begin{array}{l}\text { slopes } \\
\text { gallery }\end{array}$ & $\begin{array}{l}210-536 \\
302-582\end{array}$ & jack load & $6-26$ & $40-150$ & $5.2-3.6$ \\
\hline Speccheri & Italy & limestone & both slopes & 550 & jack load & & 600 & 1 \\
\hline $\begin{array}{l}\text { Pieve di } \\
\text { Cadore }\end{array}$ & Italy & limestone & $\begin{array}{l}\text { right slope } \\
\text { Pian delle Ere } \\
\text { left slope }\end{array}$ & $\begin{array}{r}465 \\
250-515 \\
210\end{array}$ & hydr. chamb. & 12 & 35, inj. 52 & $10-7$ \\
\hline Val Gallina & Italy & limestone & $\begin{array}{l}\text { right slope } \\
\text { left slope }\end{array}$ & $\begin{array}{l}185 \\
175\end{array}$ & hydr. chamb. & & $\begin{array}{l}50-25 \text {, inj. } 40 \\
\quad 39\end{array}$ & $\begin{array}{l}7.4-3.7 \\
4.5\end{array}$ \\
\hline Vajont & Italy & limestone & $\begin{array}{l}\text { upper slopes } \\
\text { lower slopes }\end{array}$ & $\begin{array}{l}330-460 \\
314-1400\end{array}$ & hydr. chamb. & $\begin{array}{l}24 \\
40\end{array}$ & $\begin{array}{r}40-50 \\
120\end{array}$ & $\begin{array}{c}9-8 \\
\text { up to } 11\end{array}$ \\
\hline Maë & Italy & limestone & $\begin{array}{l}\text { valley bottom } \\
\text { right slope } \\
\text { left slope }\end{array}$ & $\begin{array}{l}870 \\
310 \\
260\end{array}$ & hydr. chamb. & 20 & $\begin{array}{l}\text { 85, inj. } \\
65 \text {, inj. }\end{array}$ & $\begin{array}{l}3.7 \\
4\end{array}$ \\
\hline Fedaia & Italy & limestone & $\begin{array}{l}\text { right slope } \\
\text { left slope }\end{array}$ & $\begin{array}{l}385 \\
395\end{array}$ & hydr. chamb. & 25 & 75 , inj. & 5.2 \\
\hline
\end{tabular}

The moduli calculated from dynamic tests are generally much higher than those calculated from static tests. In seismic tests, the stress level is usually much lower than in static tests (after Link, 1964).

\section{Static vs. dynamic moduli; sedimentary rocks (cont.)}

\begin{tabular}{|c|c|c|c|c|c|c|c|c|c|c|}
\hline Well & $\begin{array}{l}\text { Depth } \\
\text { (m) }\end{array}$ & $\begin{array}{l}\text { Lab. } \\
\text { Static }\end{array}$ & $\begin{array}{c}E(\mathrm{GPa}) \\
\mathrm{Lab} . \\
\text { Dyn. }\end{array}$ & $\begin{array}{l}\text { Field } \\
\text { Dyn. }\end{array}$ & $\begin{array}{l}\text { Lab. }{ }^{b} \\
\text { Static }\end{array}$ & $\begin{array}{c}\text { J(GPa }) \\
\text { Lab. } \\
\text { Dyn. }\end{array}$ & $\begin{array}{l}\text { Field } \\
\text { Dyn. }\end{array}$ & $\begin{array}{l}\text { Lab. } \\
\text { Static }\end{array}$ & $\begin{array}{c}v \\
\text { Lab." } \\
\text { Dyn. }\end{array}$ & $\begin{array}{l}\text { Field } \\
\text { Dyn. }\end{array}$ \\
\hline PTS 24-19 & 1581.6 & 10.38 & 45.09 & 43.62 & 3.8 & 23.0 & 16.66 & 0.34 & 0.05 & 0.31 \\
\hline PTS 22-12 & 1958.0 & 16.99 & 49.50 & 29.98 & 6.57 & 25.12 & 11.08 & 0.29 & 0.024 & 0.35 \\
\hline PTS 3-10A & 3512.5 & 41.66 & 66.02 & 51.12 & 17.18 & 33.04 & 21.04 & 0.21 & 0.008 & 0.21 \\
\hline RR 1-3 & 3803.6 & 22.59 & 63.61 & 45.21 & 9.23 & 36.52 & 18.31 & 0.26 & 0.15 & 0.24 \\
\hline
\end{tabular}

"Values taken to be the average of $E_{w}, E, G, G$, or $v, v$.

'Values of laboratory static equals to $G_{x y}$ (assuming $G_{x y} \simeq G_{x z}$ ).

3-way comparison of elastic constants for the Mesaverde sandstone

(After Lin and Heuze, 1987) 


\section{Estimating joint normal stiffness in Climax granite}

For a rock mass with three orthogonal joint sets, equally spaced, the field modulus is given by (Duncan and Goodman, 1968):

$$
1 / E_{f}=1 / E_{r}+1 / s \cdot K_{n}
$$

where

$-E_{r}=$ rock material modulus

- s = joint spacing

- $K_{n}=$ normal joint stiffness

The joint spacing could be estimated from the RQD (next slide).

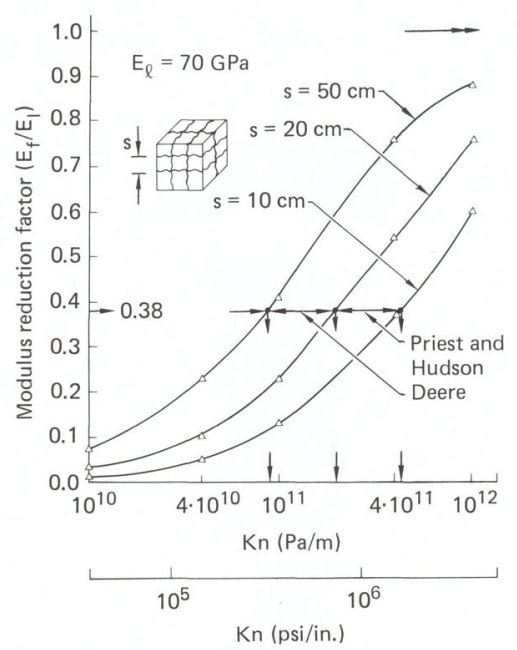

\section{Joint spacing versus RQD}

After Deere (1964), and Priest and Hudson (1976)

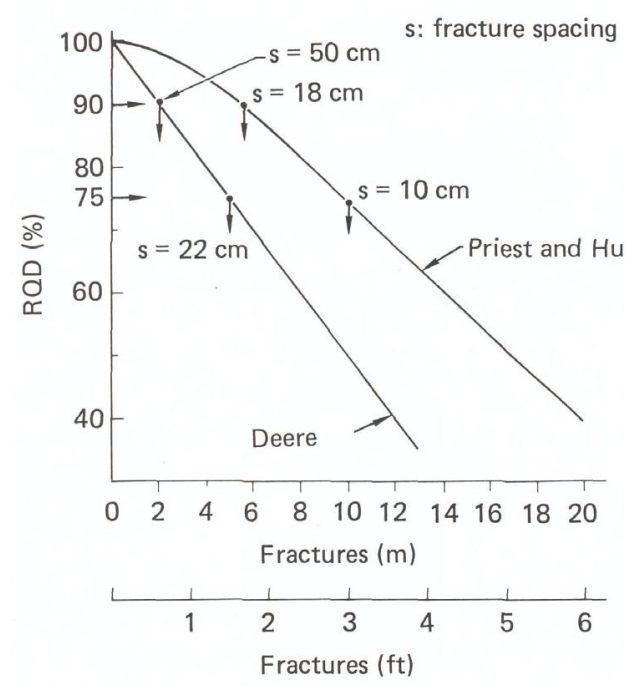




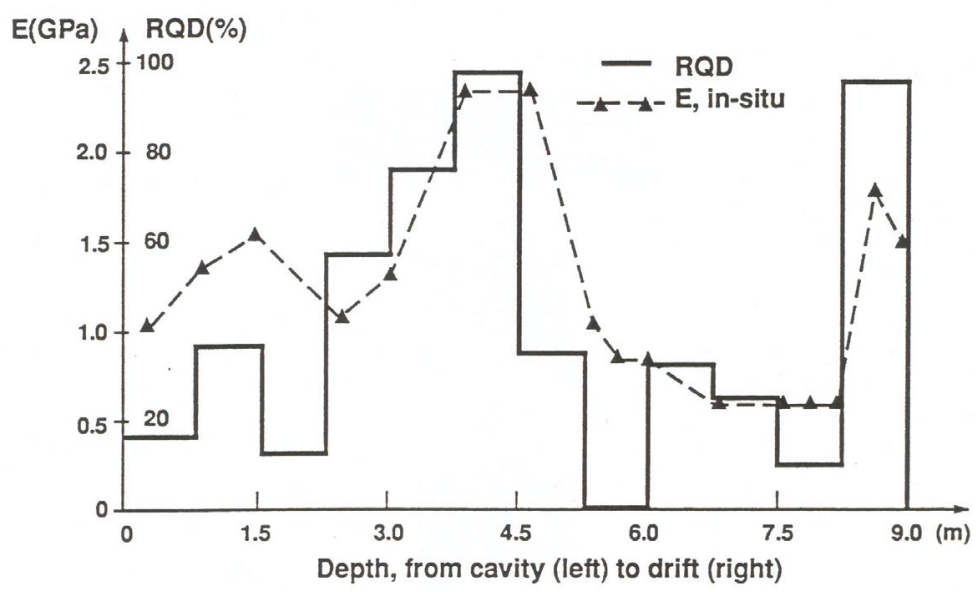

Example in tuff , Nevada Test Site, (Heuze et al., 1995)

\section{Additional models of jointed rock masses}

$$
\begin{aligned}
& E_{1}=\frac{1}{\left(\frac{1}{E_{r}}+\frac{1}{S_{1} K_{n 1}}\right)} \\
& \mathrm{G}_{12}=\frac{1}{\left(\frac{1}{\mathrm{G}_{\mathrm{r}}}+\frac{1}{\mathrm{~S}_{1} \mathrm{~K}_{\mathrm{s} 1}}+\frac{1}{\mathrm{~S}_{2} \mathrm{~K}_{\mathrm{s} 2}}\right)} \\
& v_{12}=v_{13}=v_{r} \frac{E_{1}}{E_{r}} \\
& \mathrm{E}_{2}=\frac{1}{\left(\frac{1}{\mathrm{E}_{\mathrm{I}}}+\frac{1}{\mathrm{~S}_{2} \mathrm{~K}_{\mathrm{n} 2}}\right)} \\
& \mathrm{G}_{13}=\frac{1}{\left(\frac{1}{\mathrm{G}_{\mathrm{r}}}+\frac{1}{\mathrm{~S}_{1} \mathrm{~K}_{\mathrm{s} 1}}+\frac{1}{\mathrm{~S}_{3} \mathrm{~K}_{\mathrm{s} 3}}\right)} \\
& \nu_{23}=v_{21}=\nu_{r} \frac{E_{2}}{E_{r}} \\
& v_{31}=v_{32}=v_{r} \frac{E_{3}}{E_{r}} \\
& E_{3}=\frac{1}{\left(\frac{1}{E_{r}}+\frac{1}{S_{3} K_{n 3}}\right)} \\
& G_{23}=\frac{1}{\left(\frac{1}{G_{r}}+\frac{1}{S_{2} K_{s 2}}+\frac{1}{S_{3} K_{s 3}}\right)}
\end{aligned}
$$

E's : Young's moduli; G's : shear moduli; V's ; Poisson's ratios

Three orthogonal joint sets, not equally spaced (Duncan and Goodman, 1968).

See also Gerrard (1982), and Fossum (1985) 


\section{Comparison of different tests - Scale effects}

(Wallace et al, 1972)

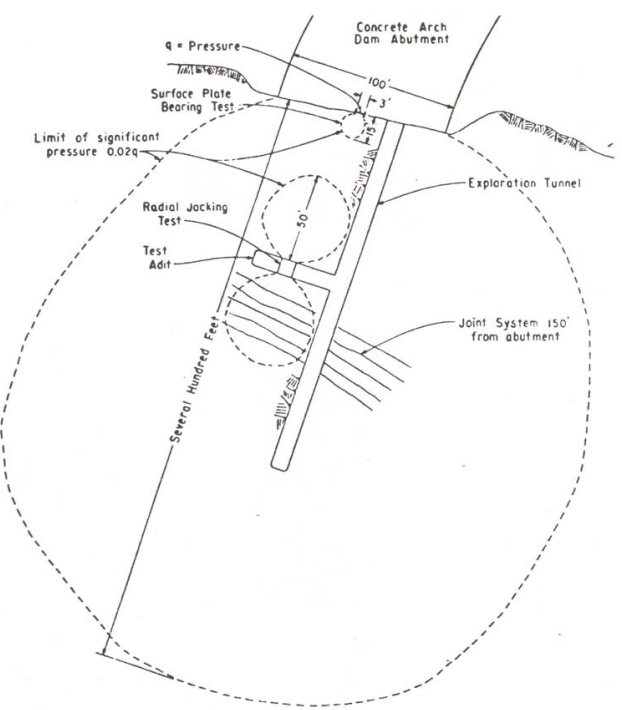

\section{Comparison of different tests - Scale effects}

\begin{tabular}{|c|c|c|}
\hline \multirow[t]{2}{*}{ Type of test } & \multicolumn{2}{|c|}{ "Test Volume" } \\
\hline & $\mathrm{dm}^{3}$ & $\mathrm{ft}^{3}$ \\
\hline \multicolumn{3}{|l|}{ Strength } \\
\hline $81 \mathrm{~cm}(32 \mathrm{in})$ diameter, 2 by 1 cylinder $\ldots \ldots \ldots \ldots \ldots$ & 835 & 30 \\
\hline 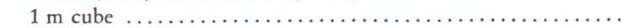 & 1000 & 35 \\
\hline $15 \times 15 \mathrm{~cm} \quad(6$ in $\times 6$ in) plate bearing $\ldots \ldots \ldots \ldots \ldots \ldots \ldots \ldots \ldots$ & 170 & 6 \\
\hline $23 \times 23 \mathrm{~cm} \quad(9 \mathrm{in} \times 9$ in) plate bearing $\ldots \ldots \ldots \ldots \ldots \ldots \ldots \ldots \ldots$ & 570 & 20 \\
\hline $30 \times 30 \mathrm{~cm}(12$ in $\times 12$ in) plate bearing $\ldots \ldots \ldots \ldots \ldots \ldots \ldots \ldots \ldots \ldots$ & 1380 & 48 \\
\hline \multicolumn{3}{|l|}{ Deformability } \\
\hline 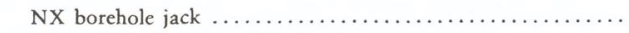 & 130 & 4.6 \\
\hline $30 \mathrm{~cm}\left(12^{\prime \prime}\right)$ diameter, plate bearing $\ldots \ldots \ldots \ldots \ldots \ldots \ldots \ldots$ & 950 & 33.6 \\
\hline $91 \mathrm{~cm}\left(36^{\prime \prime}\right)$ diameter, plate bearing $\ldots \ldots \ldots \ldots \ldots \ldots \ldots \ldots \ldots$ & 26000 & 908 \\
\hline Pressure tunnel, $1.5 \mathrm{~m}$ diameter, $6 \mathrm{~m}$ long $\ldots \ldots \ldots \ldots \ldots$ & 82000 & 1415 \\
\hline "Petite sismique" & $\begin{array}{l}\text { Up to } \\
\text { thousa }\end{array}$ & \\
\hline
\end{tabular}

These volumes are to be compared to those of the following laboratory tests:

$\begin{array}{rrr}-N X \text { sample } \ldots \ldots \ldots \ldots \ldots \ldots \ldots \ldots & 244 \mathrm{~cm}^{3} & \left(15 \mathrm{in}^{3}\right) \\ -10 \mathrm{~cm}(4 \text { in }) \text { cube } \ldots \ldots \ldots \ldots \ldots \ldots & 1000 \mathrm{~cm}^{3} & \left(64 \mathrm{in}^{3}\right) \\ -20 \mathrm{~cm}(8 \text { in) diameter, } & & \\ 2 \text { by } 1 \text { cylinder } \ldots \ldots \ldots \ldots \ldots \ldots & 0.013 \mathrm{~m}^{3} & \left(800 \mathrm{in}^{3}\right)\end{array}$

Heuze, 1980 


\section{Summary of scale effects}

Heuze, 1980

\begin{tabular}{|c|c|c|c|c|c|c|}
\hline $\begin{array}{l}\text { Name of project, } \\
\text { date and reference }\end{array}$ & Rock type & $\begin{array}{l}\text { Type of field } \\
\text { test }\end{array}$ & $\begin{array}{l}\text { No. of } \\
\text { tests }\end{array}$ & $\begin{array}{l}E_{F^{*}} \\
(\mathrm{GPa})\end{array}$ & $\begin{array}{l}E_{L^{*}} \\
(\mathrm{GPa})\end{array}$ & $E_{\boldsymbol{F}} / E_{L}$ \\
\hline $\begin{array}{l}\text { Waldeck II } \\
1973 \text { (41) }\end{array}$ & $\begin{array}{l}\text { Greywacke } \\
\text { (S) }\end{array}$ & $\begin{array}{l}\text { Plate bearing } \\
\text { Tunnel relaxation }\end{array}$ & & $\begin{array}{r}5.0 \\
15.0\end{array}$ & 20.0 & $\begin{array}{l}0.25 \\
0.75\end{array}$ \\
\hline $\begin{array}{l}\text { Mica Project } \\
1974 \text { (37) }\end{array}$ & $\begin{array}{l}\text { Quartzite } \\
\text { Gneiss (M) } \\
\text { (M) }\end{array}$ & $\begin{array}{l}\text { Plate bearing } \\
\text { Flat jacks } \\
\text { Goodman jack }\end{array}$ & $\begin{array}{r}12 \\
19 \\
132\end{array}$ & $\begin{array}{l}27.6 \\
28.8 \\
16.6\end{array}$ & 27.0 & $\begin{array}{l}1.04 \\
1.07 \\
0.61\end{array}$ \\
\hline $\begin{array}{l}\text { Channel Tunnel } \\
1975 \text { (53) }\end{array}$ & $\begin{array}{l}\text { Chalk } \\
\text { (S) }\end{array}$ & Plate bearing & & 2.4 & 0.7 & 3.42 \\
\hline $\begin{array}{l}\text { LG-2 Project } \\
1976 \text { (38) }\end{array}$ & $\begin{array}{l}\text { Massive } \\
\text { Granite (I) }\end{array}$ & Plate bearing & & 50.0 & 80.0 & 0.62 \\
\hline $\begin{array}{l}\text { Dinorwic } \\
1977 \text { (19) }\end{array}$ & $\begin{array}{l}\text { Slate } \\
(\mathrm{M})\end{array}$ & RQD index & & 50.0 & 105.0 & 0.48 \\
\hline \multirow[t]{2}{*}{$\begin{array}{l}\text { Elandsberg } \\
1977(8)\end{array}$} & $\begin{array}{l}\text { Greywake } \\
\text { (S) }\end{array}$ & $\begin{array}{l}\text { Plate bearing } \\
\text { Small flat jacks } \\
\text { Large flat jacks } \\
\text { Goodman jack } \\
\text { Tunnel relaxation } \\
\text { Petite sismique } \\
\text { RQD prediction } \\
\text { RMR prediction }\end{array}$ & $\begin{array}{l}33 \\
37 \\
3 \\
39 \\
23 \\
43 \\
34 \\
45\end{array}$ & $\begin{array}{l}39.6 \\
45.5 \\
42.2 \\
28.4 \\
42.5 \\
26.0 \\
35.5 \\
41.3\end{array}$ & 73.4 & $\begin{array}{l}0.54 \\
0.62 \\
0.57 \\
0.39 \\
0.58 \\
0.35 \\
0.48 \\
0.56\end{array}$ \\
\hline & $\begin{array}{l}\text { Phyllite } \\
\text { (M) }\end{array}$ & $\begin{array}{l}\text { Small flat jack } \\
\text { Goodman jack } \\
\text { Tunnel relaxation } \\
\text { Petite sismique } \\
\text { RQD prediction } \\
\text { RMR prediction }\end{array}$ & $\begin{array}{r}9 \\
6 \\
4 \\
25 \\
5 \\
7\end{array}$ & $\begin{array}{l}33.7 \\
12.0 \\
20.0 \\
15.4 \\
11.2 \\
20.1\end{array}$ & 56.0 & $\begin{array}{l}0.60 \\
0.21 \\
0.36 \\
0.27 \\
0.20 \\
0.36\end{array}$ \\
\hline \multirow[t]{4}{*}{$\begin{array}{l}\text { Orange River } \\
1976 \text { (8) }\end{array}$} & $\begin{array}{l}\text { Dolerite } \\
\text { (Verwoerd } \\
\text { Dam) } \\
\text { (I) }\end{array}$ & $\begin{array}{l}\text { Plate bearing } \\
\text { Pressure chamber } \\
\text { Tunnel relaxation } \\
\text { Petite Sismique }\end{array}$ & & $\begin{array}{l}25.5 \\
25.2 \\
23.5 \\
27.3\end{array}$ & & 0.36 \\
\hline & $\begin{array}{l}\text { Shale } \\
\text { (S) }\end{array}$ & $\begin{array}{l}\text { Plate bearing } \\
\text { Petite sismique }\end{array}$ & & $\begin{array}{l}12.8 \\
12.1\end{array}$ & & 0.40 \\
\hline & $\begin{array}{l}\text { Dolerite } \\
\text { (le Roux Dam) } \\
\text { (I) }\end{array}$ & $\begin{array}{l}\text { Plate bearing } \\
\text { Pressure chamber } \\
\text { Tunnel relaxation } \\
\text { Petite sismique }\end{array}$ & & $\begin{array}{l}26.0 \\
22.0 \\
31.8\end{array}$ & & 0.30 \\
\hline & $\begin{array}{l}\text { Mudstone } \\
\text { Siltstone } \\
\text { Sandstone } \\
\text { (S) }\end{array}$ & $\begin{array}{l}\text { Plate bearing } \\
\text { Pressure chamber }\end{array}$ & & $\begin{array}{l}13.0 \\
17.8 \\
10.0\end{array}$ & & 0.70 \\
\hline
\end{tabular}

\section{Summary of scale effects (cont.)}

Heuze, 1980

Ratios $E_{F} / E_{L}$ for the Three Rock Classes

\begin{tabular}{|c|c|c|c|}
\hline Rock class & $\begin{array}{l}\text { No. of } \\
\text { results }\end{array}$ & Mean & Std. Dev. \\
\hline Igneous $\ldots \ldots \ldots \ldots \ldots$ & 15 & 0.35 & 0.16 \\
\hline Metamorphics ......... & 41 & 0.36 & 0.23 \\
\hline Sedimentaries $\ldots \ldots \ldots \ldots$ & 22 & 0.42 & 0.26 \\
\hline
\end{tabular}

Ratios $E_{F} / E_{L}$ for Various Types of Field Deformability Tests

\begin{tabular}{llll} 
Type of test & $\begin{array}{l}\text { No. of } \\
\text { results }\end{array}$ & Mean & Std. Dev. \\
\hline Plate bearing $\ldots \ldots \ldots \ldots \ldots \ldots \ldots \ldots \ldots$ & 27 & 0.32 & 0.26 \\
Full scale deformation $\ldots \ldots \ldots \ldots \ldots \ldots$ & 14 & 0.44 & 0.26 \\
Flat jacks $\ldots \ldots \ldots \ldots \ldots \ldots \ldots \ldots \ldots$ & 10 & 0.54 & 0.27 \\
Borehole jack or dilatometer $\ldots \ldots \ldots \ldots$ & 9 & 0.33 & 0.17 \\
Pressure chamber $\ldots \ldots \ldots \ldots \ldots \ldots$ & 8 & 0.45 & 0.22 \\
Petite sismique $\ldots \ldots \ldots \ldots \ldots \ldots \ldots \ldots$ & 5 & 0.34 & 0.05 \\
Others $\ldots \ldots \ldots \ldots \ldots \ldots \ldots \ldots$ & 5 & 0.42 & 0.14
\end{tabular}




\section{Plate tests on bedded (anisotropic) rocks}

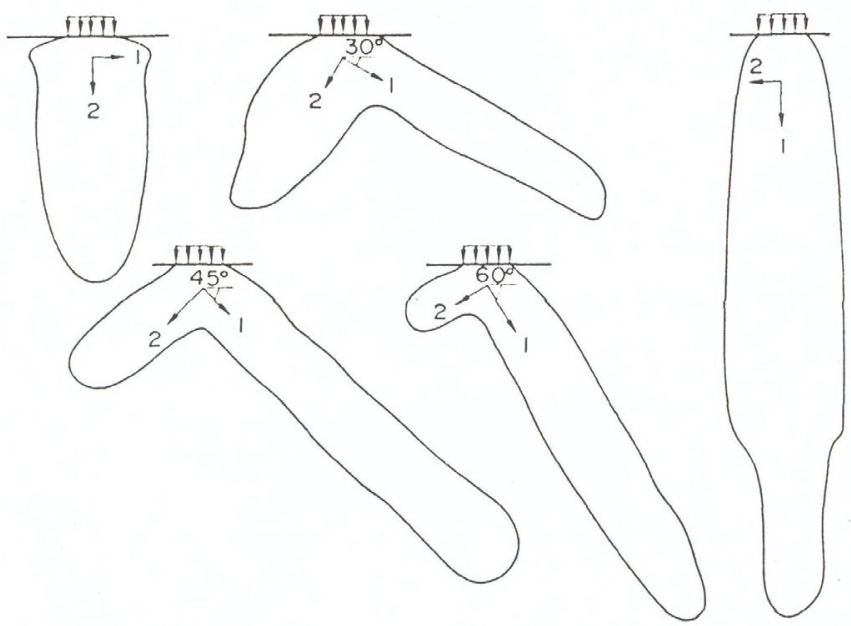

Pressure bulb shape under a plate, influenced by rock mass anisotropy (Singh, 1973a). In the figure, direction 1 is parallel to the bedding planes.

\section{Plate tests on bedded rocks (cont.)}

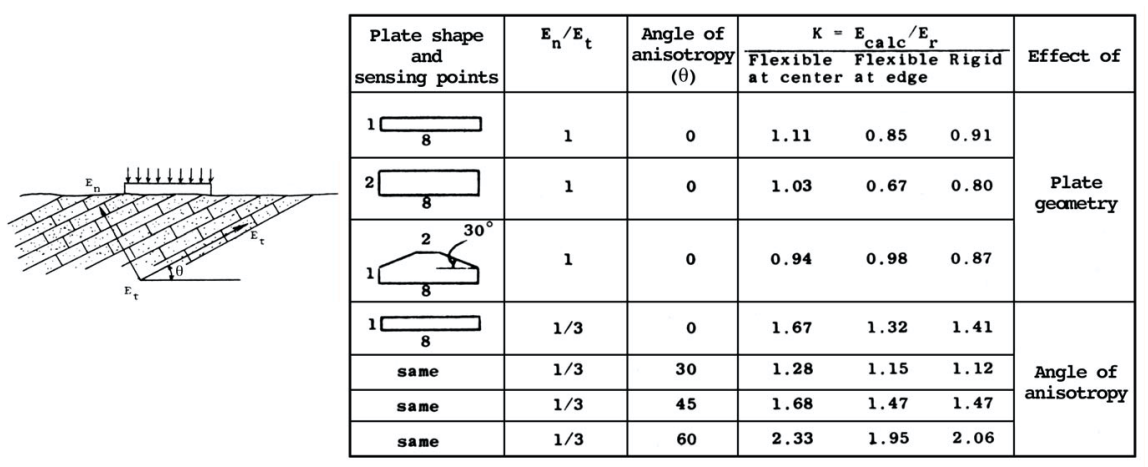

When conducting plate bearing tests on anisotropic rocks, the modulus calculated from an isotropic solution can be in error due to the rock mass anisotropy and possibly due to the plate geometry. Results based on 2-D finite element simulations (Heuze and Salem, 1977). 


\section{Strength of Rock Masses}

In-situ strength tests - Compressive strength
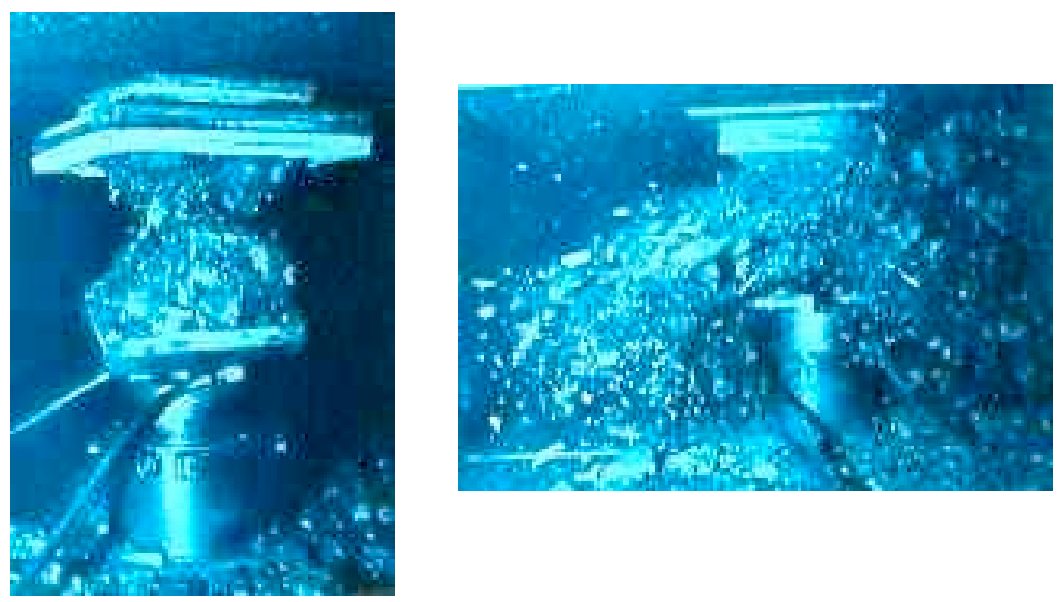

See Bieniawski and Van Herden, 1975 


\section{Compressive strength scale effects}

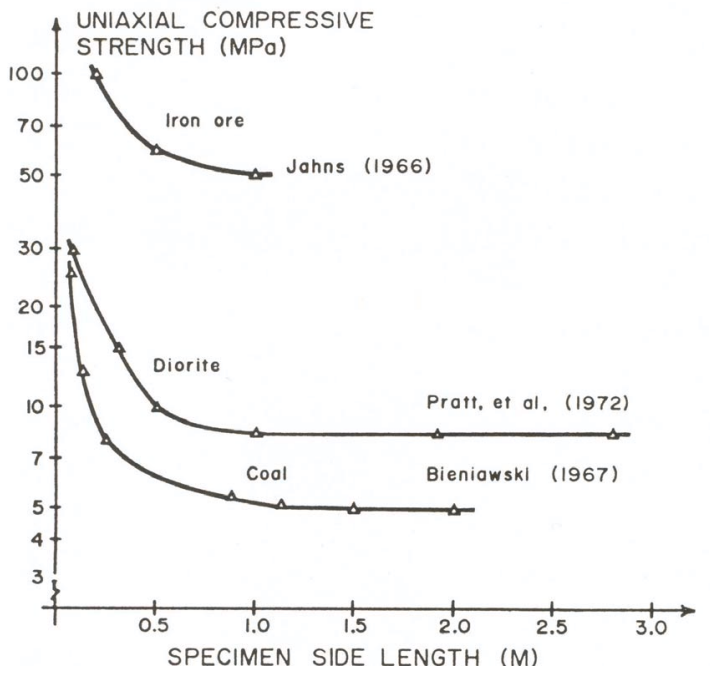

Compressive strength scale effects (cont.)

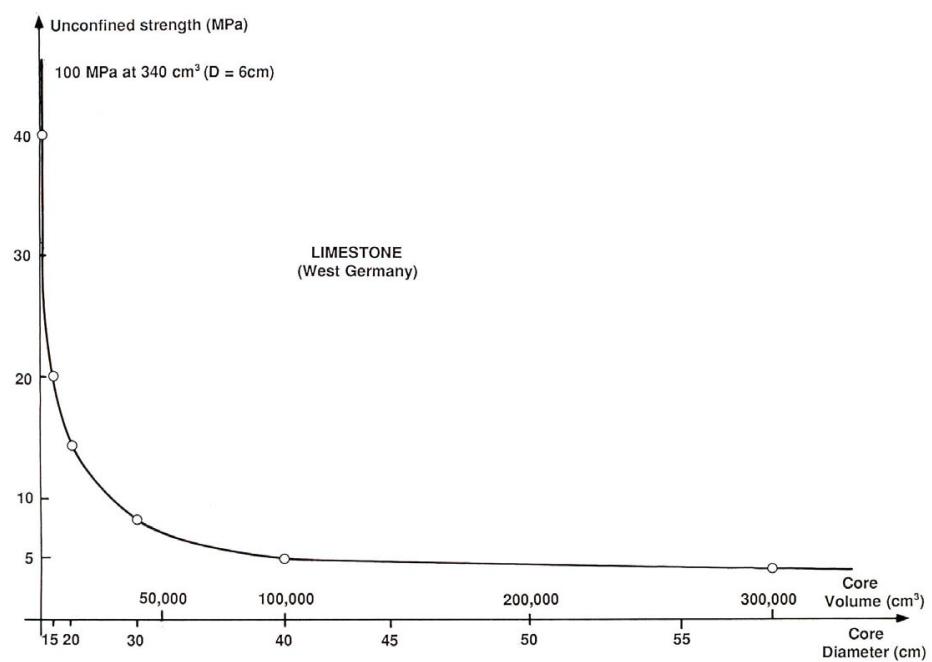




\section{The 1980 Hoek and Brown rock mass strength equation}

$$
\sigma_{1}^{\prime}=\sigma_{3}^{\prime}+\sigma_{c i}\left(m \frac{\sigma_{3}^{\prime}}{\sigma_{c i}}+s\right)^{0.5}
$$

where $\sigma_{1}^{\prime}$ and $\sigma_{3}^{\prime}$ are the major and minor effective principal stresses at failure

$\sigma_{c i}$ is the uniaxial compressive strength of the intact rock material and

$m$ and $s$ are material constants, where $s=1$ for intact rock.

The 1988 update

Hoek and Brown provided a figure indicating when to apply the rock mass criterion. Note the reference to Amadei, 1988.

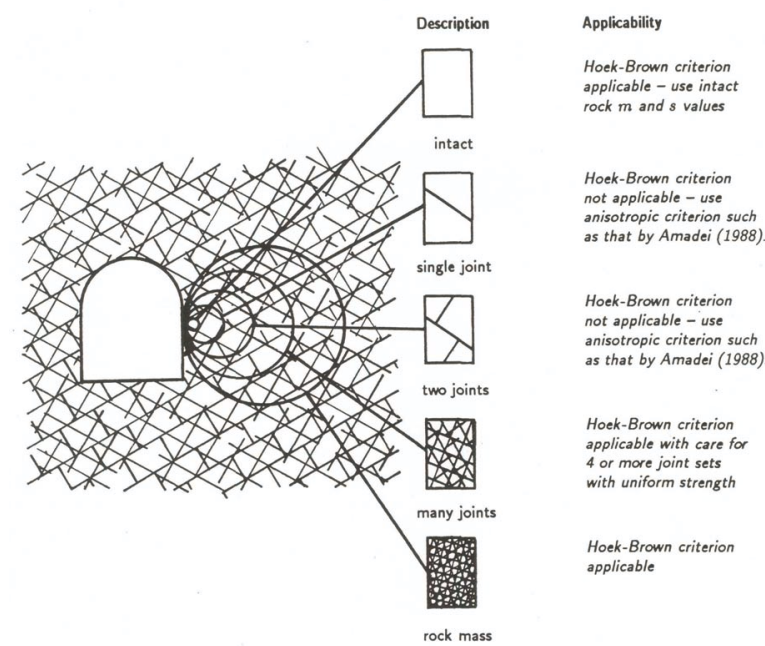


The Geological Strength Index - GSI (Hoek,1994)

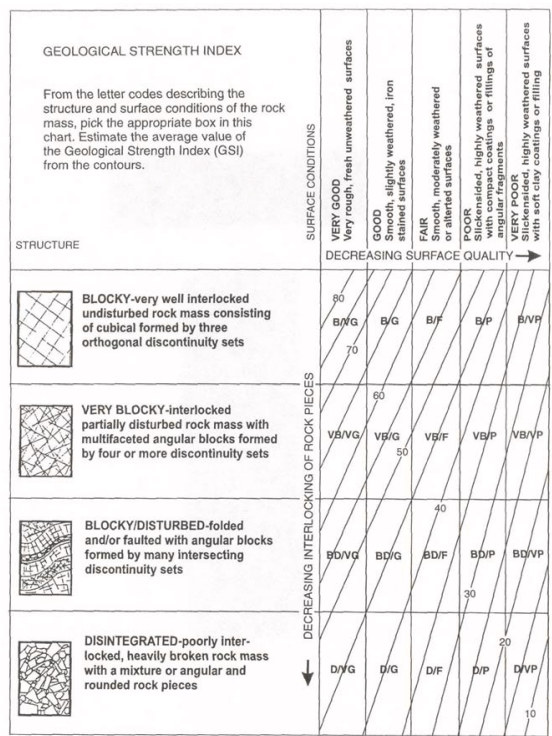

- Hoek and Brown have stated that the GSI can be obtained from the RMR of Bieniawski (1989) as follows:

$$
\text { GSI = RMR } \text { R9 }^{-5}
$$

where $\mathrm{RMR}_{\mathbf{8 9}}$ has the groundwater rating set to 15 and the adjustment for joint orientation is set to zero.

- This correlation should not be used for poor quality rock masses, i.e. with GSI $<25$. 


\section{The 2002 Update}

The entire procedure is available online at: www.rocscience.com, in the program RockLab, that includes tables and charts to estimate $\sigma_{\mathrm{ci}}, \mathrm{m}_{\mathrm{i}}$, and the GSI. The strength equations are:

$$
\sigma_{1}^{\prime}=\sigma_{3}^{\prime}+\sigma_{c i}\left(m_{b} \frac{\sigma_{3}^{\prime}}{\sigma_{c i}}+s\right)^{a}
$$

where $m_{b}$ is a reduced value of the material constant $m_{i}$ and is given by

$$
\begin{gathered}
m_{b}=m_{i} \exp \left(\frac{G S I-100}{28-14 D}\right) \\
s=\exp \left(\frac{G S I-100}{9-3 D}\right) \\
a=\frac{1}{2}+\frac{1}{6}\left(e^{-G S I / 15}-e^{-20 / 3}\right)
\end{gathered}
$$

$D$ is a factor which depends upon the degree of disturbance to which the rock mass has been subjected by blast damage and stress relaxation. It varies from 0 for undisturbed in situ rock masses to 1 for very disturbed rock masses.

\section{The $m_{i}$ coefficient}

The mi coefficient should be determined by statistical analysis of the results of a set of triaxial tests. When that is not available, the table below can be used for estimates (Hoek and Brown, 1997).

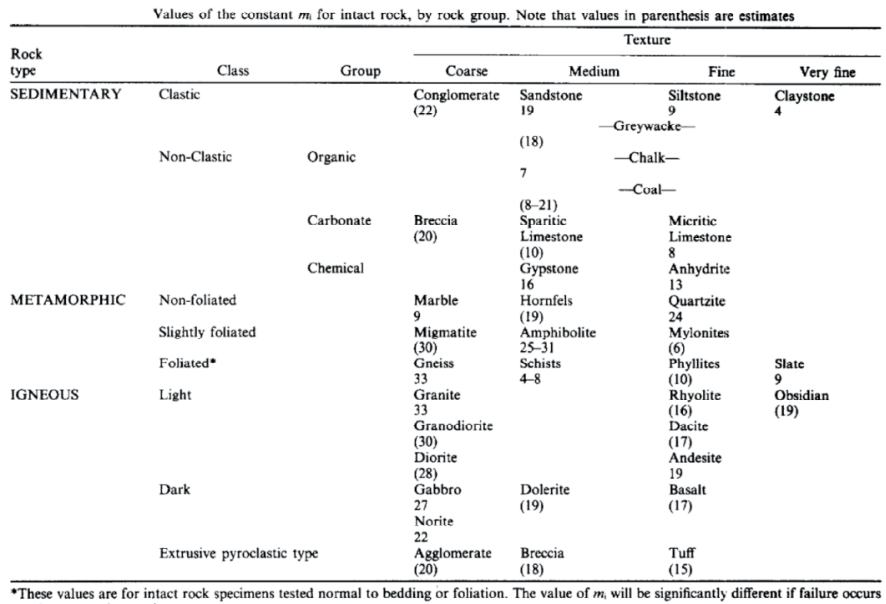




\section{The 2002 Update - The Damage factor}

\begin{tabular}{|l|l|l|}
\hline Appearance of rock mass & Description of rock mass & $\begin{array}{l}\text { Suggested } \\
\text { value of } D\end{array}$ \\
\hline & $\begin{array}{l}\text { Excellent quality controlled blasting or excavation by } \\
\text { Tunnel Boring Machine results in minimal disturbance } \\
\text { to the confined rock mass surrounding a tunnel. }\end{array}$ & $D=0$ \\
\hline & $\begin{array}{l}\text { Mechanical or hand excavation in poor quality rock } \\
\text { masses (no blasting) results in minimal disturbance to } \\
\text { the surrounding rock mass. } \\
\text { Where squeezing problems result in significant floor } \\
\text { heave, disturbance can be severe unless a temporary } \\
\text { invert, as shown in the photograph, is placed. }\end{array}$ & No invert \\
\hline
\end{tabular}

\section{The 2002 Update - The Damage factor (cont.)}

\begin{tabular}{|l|l|l|}
\hline Appearance of rock mass & Description of rock mass & $\begin{array}{l}\text { Suggested } \\
\text { value of } D\end{array}$ \\
\hline & $\begin{array}{l}\text { Small scale blasting in civil engineering slopes results } \\
\text { in modest rock mass damage, particularly if controlled } \\
\text { blasting is used as shown on the left hand side of the } \\
\text { photograph. However, stress relief results in some } \\
\text { disturbance. }\end{array}$ & $\begin{array}{l}D=0.7 \\
\text { Good blasting } \\
D=1.0 \\
\text { Poor blasting }\end{array}$ \\
\hline & $\begin{array}{l}\text { Very large open pit mine slopes suffer significant } \\
\text { disturbance due to heavy production blasting and also } \\
\text { due to stress relief from overburden removal. }\end{array}$ & $\begin{array}{c}D=1.0 \\
\text { Production } \\
\text { blasting } \\
D=0.7 \\
\text { In some softer rocks excavation can be carried out by } \\
\text { Mechanical } \\
\text { excavation }\end{array}$ \\
\hline ripping and dozing and the degree of damage to the \\
slopes is less.
\end{tabular}




$$
E_{m}(G P a)=\left(1-\frac{D}{2}\right) \sqrt{\frac{\sigma_{c i}}{100}} \cdot 10^{((G S I-10) / 40)}
$$

\section{Other, proposed correlations}

\begin{tabular}{|c|c|c|c|}
\hline Empirical equation & Required parameters & Limitations & Equation \\
\hline Bieniawski [2] & RMR & $\mathrm{RMR}>50$ & $E_{\mathrm{M}}=2 \mathrm{RMR}-100$ \\
\hline Serafim and Pereira $[4]$ & RMR & $\mathrm{RMR} \leqslant 50$ & $E_{\mathrm{M}}=10^{(\mathrm{RMR}-10) / 40}$ \\
\hline Nicholson and Bieniawski [5] & $E_{i}$ and RMR & - & $E_{\mathrm{M}}=E_{i}\left[0.0028 \mathrm{RMR}^{2}+0.9 \operatorname{cxp}(\mathrm{RMR} / 22.82]\right.$ \\
\hline Mitri et al. [6] & $E_{i}$ and $\mathrm{RMR}$ & - & $E_{\mathrm{M}}=E_{i}\left[0.5\left(1-\left(\cos \left(\pi^{*} \mathrm{RMR} / 100\right)\right)\right)\right]$ \\
\hline Hoek and Brown [7] & GSI and UCS & $\mathrm{UCS} \leqslant 100 \mathrm{MPa}$ & $E_{\mathrm{M}}=\sqrt{\frac{\mathrm{UCS}}{100}} 10^{(\mathrm{GSI}-10) / 40}$ \\
\hline Barton [1] & $Q$ & & $E_{\mathrm{M}}=10 Q_{\mathrm{c}}^{1 / 3} Q_{\mathrm{c}}=Q \frac{\mathrm{UCS}}{100}$ \\
\hline Palmström and Singh [8] & $\mathrm{RMi}$ & - & $\begin{array}{l}\mathrm{RMi}>0.1, E_{\mathrm{M}}=5.6 \mathrm{RMi}^{0.375} \\
0.1<\mathrm{RMi}<301, E_{\mathrm{M}}=7 \mathrm{RMi}^{0.4}\end{array}$ \\
\hline Kayabasi et al. [9] & $E_{i}, \mathrm{RQD}$ and WD & - & $E_{\mathrm{M}}=0.135\left[\frac{E_{i}(1+\mathrm{RQD} / 100)}{\mathrm{WD}}\right]^{1.1811}$ \\
\hline
\end{tabular}

[1] I. J. Rock Mechanics, v.39, 185-216, 2002

[2] I. J. Rock Mechanics, v. 15, 237-247, 1978

[4] Proc. Symp. Eng. Geol. Underground Openings,Lisbon, 1983

[5] I. J. Mining and Geol. Eng., v. 8, 181-202, 1990

[6] SME Annual Mtg., Albuquerque, 94-116, 1994

[7] I. J. Rock Mechanics, v. 34, n. 8, 1165-1186, 1997. Note the 2002 update, on the previous slide.

[8] Tunneling and Undergr. Space Technology, v. 16, ,115-131, 2001

[9] I. J. Rock Mechanics, v.40, 55-63, 2003 


\section{Additional strength criteria for rock masses}

- Coal seams (after Kalamaras and Bieniawski, 1993) :

$$
\begin{aligned}
& \sigma_{1} / \sigma_{c}=b\left[\sigma_{3} / \sigma_{c}\right]^{0.6}+a \\
& b=\exp [(R M R+20) / 52] \\
& a=\exp [(R M R-100) / 12]
\end{aligned}
$$

- Other criteria: see, for example, Sheorey, 1997

\section{References}

Amadei, B. (1988) "Strength of a Regularly Jointed Rock Mass Under Biaxial and Axisymmetric Loading Conditions", Int. J. of Rock Mechanics and Mining Science, v. 25, n. 1, pp. 3-13.

Bandis, S., Lumsden, A. C., and Barton, N. R. (1981) "Experimental Studies of Scale Effects on the Shear Behavior of Rock Joints", Int. J. Rock Mechanics and Mining Science, v. 18, pp. 1-21.

Barton, N. (1973) "Review of a New Shear-Strength Criterion for Rock Joints", Engineering Geology, v. 7, pp.287-332.

Barton, N., Bandis, S., and Bakhtar, K. (1985) "Strength, Deformation , and Conductivity Coupling of Rock Joints”, Int. J. Rock Mechanics and Mining Science, v. 22, n. 3, pp. 121-140.

Belin, R. A. (1959) "Observations on the Behavior of Rock When Subjected to Plate Bearing Loads", Australian Journal of Applied Sciences, v. 10 n. 4.

Bieniawski, Z. T., Van Heerden, W. L. (1975) "Significance of In-Situ Tests on Large Rock Specimens" Int. J. Rock Mechanics and Mining Sciences v 12, n 4, p 101-113.

Bieniawski, Z. T. (1989) “Engineering Rock Mass Classification”, 251p. (Wiley and Sons, New York).

Blouin, S. E. and Kaiser, J. V. Jr. (1972) “Project STARMET Ground Motion Data”, v. 1, AFWL-TR$72-68$

Deere, D. V., (1964) “Technical Description of Rock Cores for Engineering Purposes", Rock Mechanics and Engineering Geology, v. 1, n. 1, pp. 17-22. 


\section{References}

Duncan, J. M., and Goodman, R. E. (1968) "Finite Element Analyses of Slopes in Jointed Rock", U.C. Berkeley, Civil Engineering, report to U.S. Army Corps of Engineers WES, Vicksburg, MS, Contract DACW 39-67-C-0091, Report S-68-3, 277 p., February.

Fardin, N., Stephansson, O., and Jing, L. (2003) "Scale Effect on the Geometrical and Mechanical Properties of Rock Joints", Proc. 10th Congress ISRM, v. 1, pp 319-324, (South African Institute of Mining and Metallurgy, Johannesburg).

Fossum, A. F. (1985) "Effective Elastic Properties for a Randomly Jointed Rock Mass", Int. J. of Rock Mechanics and Mining Science, v. 22, n. 6, pp. 467-470.

Gerrard, C. M. (1982) "Elastic Models of Rock Masses Having One, Two, and Three Sets of Joints", Int. J. of Rock Mechanics and Mining Science , v. 19, pp.15-23.

Gokceoglu, C., Sonmez, H., and Kayabasi, A. (2003) "Predicting the Deformation Moduli of Rock Masses", Int. J. of Rock Mechanics and Mining Science, v. 40, pp. 701-710.

Goodman, R. E. (1970) "The Deformability of Joints", in Determination of the In-Situ Modulus of Deformation of Rock, ASTM STP 477 pp. 174-196 (ASTM, Philadelphia, PA).

Goodman, R. E., Van, T. K., and Heuze, F. E. (1972) "Measurement of Rock Deformability in Boreholes", Proc. 10th U. S. Symp. on Rock Mechanics, Austin, TX, May, 1968, pp. 523-555 (SME, Littleton, CO).

Goodman , R. E. (1980) "Introduction to Rock Mechanics", (Wiley and Sons, New York), 478 p.

\section{References}

Heuze, F. E. and Salem, A. (1977) "Rock Deformability Measured In-Situ - Problems and Solutions", Proc. Int. Symp. on Field Measurements in Rock Mechanics, Zurich, Switzerland, April (A.A. Balkema, Rotterdam, Netherlands).

Heuze, F. E. (1980) "Scale Effects in the Determination of Rock Mass Strength and Deformability", Rock Mechanics, v. 12, pp. 167-192.

Heuze, F. E., Patrick, W. C., Butkovich, T. R., Peterson, J. C., de la Cruz, R. V., and Voss, C. F. (1982) "Rock Mechanics Studies of Mining in the Climax Granite", Int. J. of Rock Mechanics and Mining Science, v. 19, pp 167-183, 1982.

Heuze, F. E. and Amadei, B. (1985) "The NX-Borehole Jack: A Lesson in Trials and Errors", Int. J. of Rock Mechanics and Mining Science, v.22, n. 2, pp.105-112.

Heuze, F. E., Walton, O. R., Maddix, D. M., Shaffer, R. T., and Butkovich, T. R., (1993) "Analysis of Explosions in Hard Rocks: The Power of Discrete Element Modeling", pp 387-413, in Comprehensive Rock Engineering, v. 2, (Pergamon Press, London, U.K.).

Heuze, F. E., Swift, R. P., Hill, L. R., and Barrett, W. M. (1995) "Behavior of a Steel-Liner-andBolts System Under Very High Thermal and Mechanical Loading", Int. J. of Rock Mechanics and Mining Science, v. 32, n. 7, pp. 643-673.

Hoek, E., and Brown, E. T. (1980) "Empirical Strength Criterion For Rock Masses", ASCE J. Geotechnical Engineering Division, v. 106, GT 9, pp. ‘1013-1035. 


\section{References}

Hoek, E., and Brown, E. T. (1988) "The Hoek-Brown Failure Criterion - A 1988 Update", Proc. 15th Canadian Rock Mechanics Symp., pp. 31-38, University of Toronto., J. Curran Ed.

Hoek, E. (1994) “Strength of Rock and Rock Masses”, ISRM News Journal, v. 2, n. 2 pp. 4-16.

Hoek,E., and Brown, E. T. (1997) "Practical Estimates of Rock mass Strength", Int. J. of Rock Mechanics and Mining Science, v. 34, n. 8, pp. 1165-1186.

Hoek, E., Carranza-Torres, C., and Corkum, B. (2002) "Hoek-Brown Failure Criterion - 2002 Edition", Proc. NARMS-TAC 2002, pp. 267-273, University of Toronto Press, Toronto (ISBN 0 $7727657084)$.

Kalamaras, G. S., and Bieniawski, Z. T. (1993) "A Rock Mass Strength Concept for Coal Seams", Proc. 12th Int. Conf. on Ground Control in Mining, Morgantown, WV, pp 274-283, (S. Peng Ed., University of West Virginia).

Lin, W., and Heuze, F. E. (1987) "Comparison of In-Situ Dynamic Moduli and Laboratory Moduli of Mesaverde Rocks”, Int. J. of Rock Mechanics and Mining Science, v. 24, n. 4, pp. 257-263.

Link, H. (1964) "Evaluation of Elasticity Moduli of Dam Foundation Rock Determined Seismically in Comparison to Those Arrived at Statically", Proc. 8th Int. Congress on Large Dams, v. 4, C-5, Edinburgh.

Melzer, S. (1970) “The Sugarshot Test Series”, AFWL report, 6 p.,February

\section{References}

Pratt, H. R., Black, A. D., Brown, ,W. S., and Brace, W. F. (1972) "The Effect of Specimen Size on the Mechanical Properties of Unjointed Dorite", Int. J. of Rock Mechanics and Mining Science, v. 9 , n. 4, pp. 513-529.

Priest, S. D., and Hudson, J. A. (1976) "Discontinuity Spacings in Rock", Int. J. of Rock Mechanics and Mining Science, v. 8, n. 4, pp. 135-148.

Sheorey, P. R. (1997) “Empirical Rock Failure Criteria”, 176p. (A.A. Balkema, Rotterdam).

Singh, B. (1973a) "Continuum Characterization of Jointed Rock Masses. Part 1 - The Constitutive Equations”, Int. J. of Rock Mechanics and Mining Science, v. 10, n. 4, pp. 311-335.

Wallace, G. B. , Slebir, E.J., and Anderson, F. A. (1970) "Foundation Testing for Auburn Dam", Proc. 11 th U.S. Symp. on Rock Mechanics, Berkeley, CA, June, 1969, pp. $461-498$ (SME, Littleton, CO).

Wallace, G. B. , Slebir, E.J., and Anderson, F. A. (1972) "Radial Jacking Tests for Arch Dams", Proc. 10 th U.S. Symp. on Rock Mechanics, Austin, TX, May 1968, pp. 633-660 (SME, Littleton, $\mathrm{CO})$.

Walton. O. R., Maddix, D. M., Butkovich, T. R., and Heuze F. E. (1991) "Redirection of Dynamic Compressive Waves in Materials with Nearly Orthogonal and Random Joint Sets", ASME AMD vol.117, pp 65-72. 


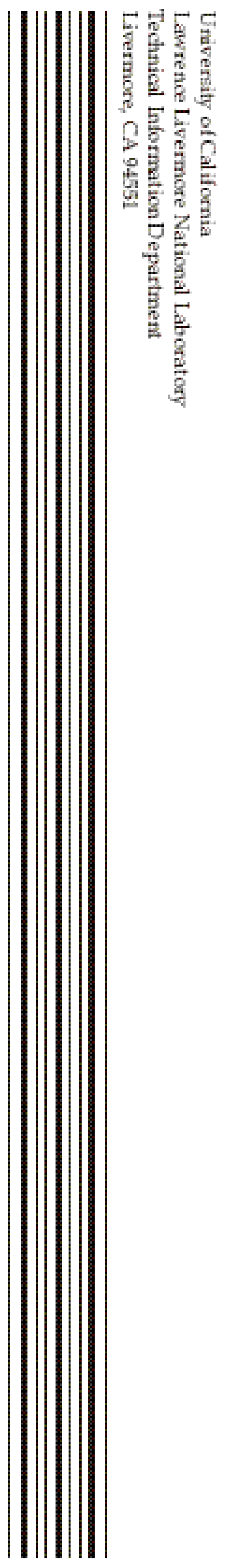

